ARTICLE

DOI: $10.1038 / s 41467-017-01150-x$

\title{
Earliest accumulation of $\beta$-amyloid occurs within the default-mode network and concurrently affects brain connectivity
}

\author{
Sebastian Palmqvist1,2, Michael Schöll (10) 1,3, Olof Strandberg ${ }^{1}$, Niklas Mattsson ${ }^{1,2}$, Erik Stomrud ${ }^{1,4}$, \\ Henrik Zetterberg ${ }^{5,6,7}$, Kaj Blennow ${ }^{5,6}$, Susan Landau ${ }^{8}$, William Jagust ${ }^{8}$ \& Oskar Hansson ${ }^{1,4}$
}

It is not known exactly where amyloid- $\beta(A \beta)$ fibrils begin to accumulate in individuals with Alzheimer's disease (AD). Recently, we showed that abnormal levels of $A \beta 42$ in cerebrospinal fluid (CSF) can be detected before abnormal amyloid can be detected using PET in individuals with preclinical AD. Using these approaches, here we identify the earliest preclinical $A D$ stage in subjects from the ADNI and BioFINDER cohorts. We show that $A \beta$ accumulation preferentially starts in the precuneus, medial orbitofrontal, and posterior cingulate cortices, i.e., several of the core regions of the default mode network (DMN). This early pattern of $A \beta$ accumulation is already evident in individuals with normal $A \beta 42$ in the CSF and normal amyloid PET who subsequently convert to having abnormal CSF A $\beta 42$. The earliest $A \beta$ accumulation is further associated with hypoconnectivity within the $D M N$ and between the DMN and the frontoparietal network, but not with brain atrophy or glucose hypometabolism. Our results suggest that $A \beta$ fibrils start to accumulate predominantly within certain parts of the DMN in preclinical $A D$ and already then affect brain connectivity.

\footnotetext{
${ }^{1}$ Clinical Memory Research Unit, Department of Clinical Sciences, Malmö, Lund University, Lund, Sweden. ${ }^{2}$ Department of Neurology, Skåne University Hospital, Lund, Sweden. ${ }^{3}$ Wallenberg Centre for Molecular and Translational Medicine and the Department of Psychiatry and Neurochemistry, University of Gothenburg, Gothenburg, Sweden. ${ }^{4}$ Memory Clinic, Skåne University Hospital, Malmö, Sweden. ${ }^{5}$ Department of Psychiatry and Neurochemstry, Institute of Neuroscience and Physiology, the Sahlgrenska Academy at the University of Gothenburg, Mölndal, Sweden. ${ }^{6}$ Clinical Neurochemistry Laboratory,

Sahlgrenska University Hospital, Mölndal, Sweden. ${ }^{7}$ Department of Molecular Neuroscience, UCL Institute of Neurology, Queen Square, London, UK. ${ }^{8}$ Helen Wills Neuroscience Institute, University of California, Berkeley, USA. Sebastian Palmqvist and Michael Schöll contributed equally to this work.

Correspondence and requests for materials should be addressed to S.P. (email: sebastian.palmqvist@med.lu.se) or to O.H. (email: oskar.hansson@med.lu.se)
} 
ccumulation of aggregated amyloid- $\beta(A \beta)$ in the brain is believed to be the first pathological mechanism of Alzheimer's disease $(\mathrm{AD})^{1}$. Previous studies have shown that cerebral deposition of $\mathrm{A} \beta$ fibrils can occur for decades before an individual with $\mathrm{AD}$ reaches the dementia stage ${ }^{2}$. The widespread cerebral distribution of $\mathrm{A} \beta$ in later $\mathrm{AD}$ stages is wellestablished $^{3,4}$, however it has been difficult to accurately identify the initial brain regions of $A \beta$ accumulation due to the long time-lag between the start of the pathophysiology and onset of symptoms.

A neuropathological staging of different $\mathrm{A} \beta$ phases in $\mathrm{AD}$ has been proposed based on post-mortem examinations and it has been shown that the first phase is characterized by $\mathrm{A} \beta$ deposits throughout the frontal, parietal, temporal, and occipital neocortex ${ }^{5}$. Studies employing positron emission tomography (PET) to visualize fibrillar $A \beta$ deposits have also attempted to identify early $A \beta$-accumulating regions. For example, when analyzing the earliest data from $\mathrm{AD}$ mutation-carriers, most cortical areas except for the sensorimotor cortex showed $A \beta$ accumulation as early as 15 years before the expected onset of symptoms ${ }^{6}$. A shortcoming of such studies is the cross-sectional design, which precludes conclusions about longitudinal events. Another issue is that it has previously been difficult to identify the earliest preclinical AD cases; when mixing such cases with later asymptomatic $\mathrm{AD}$ cases that have been accumulating $\mathrm{A} \beta$ fibrils for years, the cortical sites of accumulation of $A \beta$ identified are widespread.

In the present study, we applied a novel methodology of combining data obtained regarding $A \beta$ levels in the brain via PET, and levels of the A $\beta 42$ peptide in cerebrospinal fluid (CSF), to identify individuals who have just recently started to accumulate $A \beta$ in the brain. Previous cross-sectional studies indicate that $\mathrm{A} \beta 42$ levels decrease in the CSF as $\mathrm{A} \beta$ starts to accumulate, before fibrillar plaques can be detected with $\mathrm{PET}^{7-11}$. Recently, this was confirmed longitudinally in a cohort of more than 400 non-demented individuals where we found that those with abnormally low CSF levels of $\mathrm{A} \beta 42$, but with still normal $\mathrm{A} \beta$ levels detected by PET (CSF+/PET-), accumulated A $\beta$ fibrils longitudinally at a similar rate as those who had both abnormal CSF levels of $\mathrm{A} \beta 42$ and abnormal levels of $\mathrm{A} \beta$ detected by PET $(\mathrm{CSF}+\mathrm{PET}+)$ and four times higher than those with normal biomarker results on both parameters (CSF-/PET- $)^{12}$. This indicates that individuals with $\mathrm{AD}$ may first be defined as being $\mathrm{CSF}+/ \mathrm{PET}-$, and then progress to $\mathrm{CSF}+\mathrm{PET}+$. It might therefore be possible to identify the earliest stages of preclinical $\mathrm{AD}$ by

\section{Table 1 Baseline characteristics of the ADNI cohort}

\begin{tabular}{|c|c|c|c|c|c|}
\hline & Entire ADNI sample & $\begin{array}{l}\text { A } \\
\text { CSF-/PET- }\end{array}$ & $\begin{array}{l}\text { B } \\
\text { CSF+/PET- }\end{array}$ & $\begin{array}{l}\mathrm{C} \\
\mathrm{CSF}+/ \mathrm{PET}+\end{array}$ & $p$-value \\
\hline N & 473 & 218 & 59 & 191 & \\
\hline Baseline $\mathrm{CN} / \mathrm{MCl}$ & $37 \% / 63 \%$ & $47 \% / 53 \%$ & $44 \% / 56 \%$ & $23 \% / 77 \%$ & $\begin{array}{l}\text { A-B }=0.58 \\
\mathbf{B}-\mathbf{C}<\mathbf{0 . 0 0 1} \\
\mathbf{A}-\mathbf{C}<\mathbf{0 . 0 0 1}\end{array}$ \\
\hline Age (years) & $72.0(7.0)$ & $71.0(7.0)$ & $71.2(8.1)$ & $73.2(6.3)$ & $\begin{array}{l}A-B=0.99 \\
B-C=0.20 \\
\mathbf{A}-\mathbf{C}<\mathbf{0 . 0 0 1}\end{array}$ \\
\hline Gender (women) & $47 \%$ & $47 \%$ & $46 \%$ & $48 \%$ & 0.95 \\
\hline Education (years) & $16.5(2.6)$ & $16.7(2.6)$ & $17.0(2.2)$ & $16.1(2.6)$ & $\begin{array}{l}A-B=0.47 \\
\mathbf{B}-\mathbf{C}=\mathbf{0 . 0 0 7} \\
\mathbf{A}-\mathbf{C}=\mathbf{0 . 0 3}\end{array}$ \\
\hline Presence of the $A P O E \varepsilon 4$ allele & $40 \%$ & $16 \%$ & $46 \%$ & $65 \%$ & $\begin{array}{l}\text { A-B }<0.001 \\
\text { B-C }=0.004 \\
\text { A-C }<0.001\end{array}$ \\
\hline MMSE (0-30 points) & $28.4(1.7)$ & $28.8(1.4)$ & $28.8(1.3)$ & $27.9(1.8)$ & $\begin{array}{l}A-B=0.95 \\
\mathbf{B}-\mathbf{C}=\mathbf{0 . 0 0 1} \\
\mathbf{A}-\mathbf{C}<\mathbf{0 . 0 0 1}\end{array}$ \\
\hline $\begin{array}{l}\text { Baseline neocortical composite florbetapir } \\
\text { SUVR }^{1}\end{array}$ & $0.90(0.21)$ & $0.74(0.05)$ & $0.78(0.06)$ & $0.93(0.09)$ & $\begin{array}{l}\text { A-B }<0.001 \\
\text { B-C }<0.001 \\
\text { A-C }<0.001\end{array}$ \\
\hline Years between florbetapir PET scans (range) & $2.0(0.9-4.1)$ & $2.0(0.9-4.1)$ & $2.0(1.9-4.1)$ & $2.0(1.7-3.0)$ & 1.0 \\
\hline Years between FDG PET scans (range) & $2.0(0.8-3.1)$ & $2.0(0.8-2.7)$ & $2.1(1.9-3.1)$ & $2.0(1.8-3.0)$ & 0.72 \\
\hline Years between MRI scans (range) & $2.0(0.4-4.1)$ & $2.0(0.4-4.1)$ & $2.0(0.5-4.1)$ & $2.0(0.6-3.1)$ & 0.92 \\
\hline Hippocampus volume $\left(\mathrm{cm}^{3}\right)$ & $7.2(1.1)$ & $7.5(1.0)$ & $7.5(0.9)$ & $6.9(1.3)$ & $\begin{array}{l}\text { A-B }=0.92 \\
\text { B-C }<\mathbf{0 . 0 0 1} \\
\mathbf{A}-\mathbf{C}<\mathbf{0 . 0 0 1}\end{array}$ \\
\hline CSF A $\beta 42(n g / L)$ & $186(52)$ & $234(27)$ & $165(23)$ & $136(22)$ & $\begin{array}{l}\text { A-B }<0.001 \\
\text { B-C }<0.001 \\
\text { A-C }<0.001\end{array}$ \\
\hline CSF T-tau (ng/L) & $79(46)$ & $59(26)$ & $60(31)$ & $109(53)$ & $\begin{array}{l}A-B=0.95 \\
\mathbf{B}-\mathbf{C}<\mathbf{0 . 0 0 1} \\
\mathbf{A}-\mathbf{C}<\mathbf{0 . 0 0 1}\end{array}$ \\
\hline CSF P-tau (ng/L) & $39(23)$ & $28(12)$ & $30(26)$ & $54(25)$ & $\begin{array}{l}A-B=0.19 \\
\mathbf{B}-\mathbf{C}<\mathbf{0 . 0 0 1} \\
\mathbf{A}-\mathbf{C}<\mathbf{0 . 0 0 1}\end{array}$ \\
\hline
\end{tabular}

CN cognitively normal, CSF cerebrospinal fluid, CSF+/- abnormal/normal CSF A 442 levels, MCI mild cognitive impairment, MMSE Mini Mental State Examination, $N$ number of subjects, PET+/abnormal/normal florbetapir SUVR, SD standard deviation, SUVR standardized uptake value ratio (using a composite reference region) corrected for partial volume errors. Comparison between nonaccumulators (CSF-/PET-), early A $\beta$ accumulators (CSF+/PET-), and late A $\beta$ accumulators (CSF+/PET+). Values are given in mean (SD) if not otherwise specified. Groups were compared with Mann-Whitney $U$ statistics if significant after the Kruskal-Wallis test. Significant $p$ values are in bold. The CSF-/PET+group was not included in this table as a separate group since they were not used in any analysis and they were also too few to be used in the group comparisons $(n=5)$ 
studying individuals with abnormal CSF $A \beta 42$ and normal $A \beta$ PET (CSF+/PET-, also referred to as early A $\beta$ accumulators to indicate their stage in the accumulation of $A \beta$ ).

The main hypothesis in the present study was that $A \beta$ fibrils are more prone to start aggregating in certain regions of the brain before they can be found throughout the neocortex, and before neurodegeneration is present. Identifying these regions and examining their properties can provide important information about why $\mathrm{A} \beta$ aggregation starts in sporadic $\mathrm{AD}$, which is a crucial step for developing disease-modifying drugs. The aim of the study was therefore to identify such regions by comparing the longitudinal fibrillar $A \beta$ deposition rates in different brain regions of early $\mathrm{A} \beta$ accumulators (termed $\mathrm{CSF}+$ PET-) with non-accumulators (termed CSF-/PET-). To avoid bias from non-optimal cut-offs for CSF A $\beta 42$ levels, we also examined the correlation between continuous CSF A $\beta 42$ levels and the regional A $\beta$ PET signal in PET negative subjects. We performed the analyses in 68 predefined anatomical regions as well as employing voxelwise statistics. The analyses were performed both in a main cohort and an independent validation cohort. As the main cohort, we used the North American Alzheimer's Disease Neuroimaging Initiative (ADNI) and included all non-demented participants with CSF A $\beta 42$ and longitudinal A $\beta$ PET data (473 individuals). The Swedish BioFINDER study was used as the validation cohort, from which we included all non-demented participants with baseline CSF A $\beta 42$ and A $\beta$ PET data (406 individuals).

We also examined early regional $A \beta$ accumulation in the presumably earliest $A \beta$ accumulators; those with normal CSF $A \beta 42$ and normal $A \beta$ PET at baseline who converted to abnormal CSF A $\beta 42$ levels at follow-up but still had a normal $A \beta$ levels identified by PET scan (CSF-/PET to CSF+/PET-). Consistently, using different cohorts and methods, we identified a specific set of early $\mathrm{A} \beta$-accumulating regions, which largely corresponded to a functional brain network, the default mode network (DMN). The relationship between early stage $A \beta$ accumulation and wholebrain functional connectivity was examined in detail and revealed that hypoconnectivity, especially within the DMN and between DMN and the frontoparietal network, was associated with low CSF A $\beta 42$ levels in individuals that still had normal A $\beta$ PET scans.
To test the hypothesis that early $\mathrm{A} \beta$ accumulation is an upstream mechanism that precedes overtly decreased energy metabolism and cell death, we compared the longitudinal changes in ${ }^{18} \mathrm{~F}$-fluorodeoxyglucose (FDG) PET and volumetric magnetic resonance imaging (MRI) in the different groups. Finally, we examined the validity of using CSF/PET groups to stratify $A \beta$ stages by comparing early $(\mathrm{CSF}+\mathrm{PET}-)$ and late $(\mathrm{CSF}+\mathrm{PET}+)$ accumulators to identify known late regions of $A \beta$ accumulation, including the sensorimotor cortex.

\section{Results}

Experimental outline and baseline characteristics. We first analyzed the longitudinal results from the ADNI cohort, then replicated the result in the BioFINDER cohort and finally analyzed functional connectivity in BioFINDER.

Baseline characteristics of the ADNI sample and the different CSF/PET groups are provided in Table 1. Early stage A $\beta$ accumulators (CSF+/PET-) had significantly higher prevalence of the $A P O E \& 4$ genotype and a slightly higher neocortical florbetapir standardized uptake value ratio (SUVR), but similar baseline cognition, hippocampal volume, and CSF tau levels as non-accumulators (CSF-/PET-) (Table 1). By contrast, the nondemented late stage $\mathrm{A} \beta$ accumulators $(\mathrm{CSF}+\mathrm{PET}+$ ) showed, at baseline, signs of neurodegeneration with hippocampal atrophy, higher CSF tau levels and worse cognition, and had per definition significantly increased florbetapir SUVRs (Table 1).

Identifying early $\mathbf{A} \beta$-accumulating regions. To identify early $A \beta$ accumulation regions, longitudinal voxelwise comparisons of accumulation rates (SUVR/year) were performed between CSF +/PET- (early A $\beta$ accumulators) and CSF-/PET- (non-accumulators). The results are shown in Fig. 1a (see Supplementary Fig. 1 for whole-brain axial images). The brain regions showing significant increases in A $\beta$ PET signal over time in CSF+/PETcases included the medial orbitofrontal cortex, anterior cingulate cortex, posterior, and isthmus cingulate cortex, precuneus and to a lesser extent temporal regions. Similar significant regions were identified by correlating CSF A $\beta 42$ levels with the accumulation rates in PET- subjects to remove potential biases from the A $\beta 42$ cut-off (Fig. 1b). In the region of interest (ROI)-based

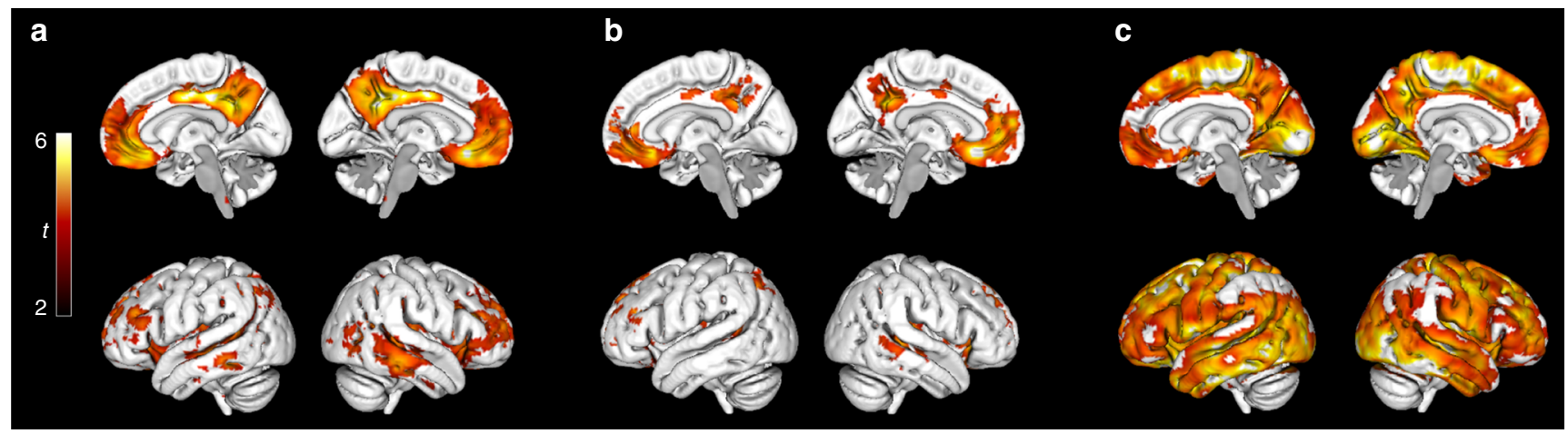

Fig. 1 Regions of $A \beta$ accumulation from longitudinal voxelwise analyses in ADNI. a shows the regions where $A \beta$ fibrils start to accumulate by comparing the annual florbetapir SUVR rate during 2 years between early stage A $\beta$ accumulators (CSF+/PET-, $n=59)$ and non-accumulators $(C S F-/$ PET-, $n=218$ ). The lateral and medial projections in a show that the most significantly increased accumulation rate among the early $A \beta$ accumulators was located in the posterior cingulate cortex, the precuneus, and the medial orbitofrontal cortex. A PET-data-derived ROI of these early-accumulating A $\beta$ regions is available at http://biofinder.se $\mathbf{b}$ confirms the regions in a without biases from a specific CSF A $\beta 42$ cut-off. Here, we performed voxelwise correlations between annual florbetapir SUVR rates and CSF A $\beta 42$ levels in A $\beta$ PET negative individuals $(n=277)$. To contrast the early stage $A \beta$ regions, $\mathbf{c}$ shows the regions with significantly increased annual SUVR rate in late stage $A \beta$ accumulators (CSF+/PET+, $n=191$ ) compared with non-accumulators (CSF-/PET-). A widespread pattern of $A \beta$ accumulation is seen in these non-demented CSF+/PET+ subjects. Voxelwise two-sample $t$-tests were used and all analyses in a-c are adjusted for age and gender. The significant threshold was set at $p<0.001$. The red and yellow colors illustrate significant $t$ values according to the scale on the left 
Table 2 Annual $\mathbf{A} \beta$ accumulation rates in the significant early $\mathbf{A} \beta$ regions in ADNI

\begin{tabular}{llll} 
Region & CSF+/PET- & CSF-/PET- & p-value \\
\hline Posterior cingulate cortex, right & $2.7 \%(1.8-3.6)$ & $0.9 \%(0.5-1.3)$ & 0.000051 \\
Medial orbitofrontal cortex, left & $2.5 \%(1.4-3.6)$ & $0.4 \%(-0.2-1.0)$ & 0.00026 \\
Medial orbitofrontal cortex, right & $2.5 \%(1.3-3.7)$ & $0.4 \%(-0.1-1.0)$ & 0.00038 \\
Precuneus, left & $2.3 \%(1.4-3.3)$ & $0.7 \%(0.2-1.2)$ & 0.00051 \\
Rostral anterior cingulate cortex, right & $2.0 \%(1.3-2.8)$ & $0.5 \%(0.0-1.0)$ & 0.00081 \\
Lateral orbitofrontal cortex, left & $1.8 \%(0.9-2.7)$ & $0.3 \%(-0.1-0.7)$ & 0.0011 \\
Insula, right & $1.8 \%(0.8-2.8)$ & $0.2 \%(-0.3-0.7)$ & 0.0025 \\
Isthmus cingulate cortex, left & $2.0 \%(1.1-2.8)$ & $0.5 \%(0.0-1.0)$ & 0.0027 \\
Rostral anterior cingulate cortex, left & $1.9 \%(1.1-2.6)$ & $0.6 \%(0.1-1.1)$ & 0.0031 \\
Isthmus cingulate cortex, right & $2.1 \%(1.2-2.9)$ & $0.5 \%(0.0-1.0)$ & 0.0032 \\
Precuneus, right & $1.9 \%(1.1-2.8)$ & $0.6 \%(0.2-1.1)$ & 0.0041 \\
Superior frontal cortex, left & $1.2 \%(0.5-2.0)$ & $0.2 \%(-0.2-0.5)$ & 0.0053 \\
Transverse temporal gyrus, right & $0.8 \%(-0.3-1.9)$ & $-0.8 \%(-1.5--0.2)$ & 0.0074 \\
Insula, left & $1.5 \%(0.7-2.3)$ & $0.2 \%(-0.3-0.7)$ & 0.0085 \\
Posterior cingulate cortex, left & $2.1 \%(1.2-3.0)$ & $0.9 \%(0.5-1.4)$ & 0.01
\end{tabular}

$\mathrm{CSF}+$ cerebrospinal fluid $\mathrm{A} \beta<192 \mathrm{ng} / \mathrm{L}, \mathrm{PET}+>0.87 \mathrm{SUVR}$, ROI region of interest, SUVR standardized uptake value ratio (using a composite reference region). Comparisons in ADNI between early A accumulators (CSF+/PET-) and non-accumulators (CSF-/PET-) subjects using general linear models with the A $\beta$ PET SUVR change/year in each of the 68 cortical Freesurfer ROI:s as the dependent variable and CSF/PET groups, sex, age, and time between PET scans as covariates. Data are given in mean values ( $95 \% \mathrm{Cl}$ of the mean) in order of significance and $\mathrm{A} \beta$ accumulation is shown in $\%$ yearly SUVR change. Only the 15 significant regions after the Benjamini \& Hochberg correction are shown. These regions were also significant when adjusting for changes in cortical thickness over 2 years (in each region) and diagnosis ( $\mathrm{MCl}$ or healthy control)

comparisons, 15 of 68 cortical regions had an increased rate in the CSF+/PET- subjects compared to the CSF-/PET- subjects (Table 2 and Supplementary Movie 1). The significant differences were in agreement with the voxelwise analyses and were mainly seen in the orbitofrontal cortex, precuneus, insula, and the posterior/isthmus cingulate cortex. On average the yearly $\mathrm{A} \beta$ accumulation rate of the $\mathrm{CSF}+\mathrm{PET}-$ group was $\sim 4-5$ times that of the CSF-/PET- group in the significant brain regions (Table 2). All results were corrected for multiple comparisons and adjusted for gender, age, and time between PET scans. To account for atrophy as a confounding factor, we also adjusted all significant models for the annual change in cortical thickness of each ROI. After this adjustment, all 15 regions still differed significantly between the two groups. The 15 early $\mathrm{A} \beta$ regions were also significant when adjusting for diagnosis (MCI or healthy control). Finally, we adjusted for $A P O E$ genotype to examine if different early $\mathrm{A} \beta$ patterns were observed depending on whether one carried an APOE $\varepsilon 4$ allele, but the same regions as in Table 2 were found (details shown in Supplementary Table 1). The distinct pattern of early $A \beta$ accumulation (Fig. 1a, b, Table 2) can be contrasted to the more global $A \beta$ accumulation pattern seen in late, but still non-demented, A $\beta$ accumulators (CSF+/PET+) compared with non-accumulators (CSF-/PET-) (Fig. 1c).

Early A $\beta$ accumulating regions in CSF converters. Previous studies have found that non-demented CSF-/PET- individuals, that during follow-up show abnormal CSF A $\beta 42$ levels, might represent the earliest preclinical $\mathrm{AD}$ phase ${ }^{13,14}$. We therefore examined CSF-/PET- subjects who, after 2 years, converted to $\mathrm{CSF}+\mathrm{PET}-$ (referred to as CSF converters). In the ADNI cohort there were $112 \mathrm{CSF}-/ \mathrm{PET}$ - subjects with longitudinal CSF data that could be included in this analysis. At follow-up investigation, 11 of these 112 subjects had converted to abnormal CSF A $\beta 42$ levels (CSF+/PET-) while 101 subjects remained stable with normal CSF A $\beta 42$ levels (CSF-/PET-). Significant differences in $\mathrm{A} \beta$ accumulation rate (A $\beta$ PET SUVR/year) between the two groups were seen in the left posterior cingulate and right medial orbitofrontal cortex $(p=0.02-0.03)$. The group comparisons were still significant after adjustment of age, gender, and time between PET scans, but probably because of the limited statistical power $(n=11)$ there were no significant differences after multiple comparison correction. Given the agreement with the previously identified regions (Fig. 1a, b and Table 2), we interpreted the identified regions as true and not a statistical type 1 error. For the CSF converters, the SUVR increased significantly with mean rates of $+2.3-2.5 \%$ per year in the left posterior cingulate cortex and the right medial orbitofrontal cortex. No significant increase was seen in these regions among the non-converters and the corresponding mean rates were $+0.3 \%$ (95\% CI -0.24 to $+0.90 \%)$ and $+0.3 \%(95 \% \mathrm{CI}-0.7$ to $+1.2 \%)$.

A $\beta$ accumulation and functional brain networks. The regional distribution of the early accumulation of cortical $A \beta$ fibrils (Fig. 1a, b) resembled the brain regions involved in the DMN. This led us to examine early accumulation of $A \beta$ fibrils within the different functional brain networks. We applied the early accumulation regions from Fig. 1a to a functional connectivity network atlas ${ }^{15}$ in order to determine the involvement of the different networks in early $A \beta$ accumulation. Figure 2 shows that early $\mathrm{A} \beta$ accumulation predominantly overlapped with the DMN (Jaccard coefficient, JC, 0.14), but also with the frontoparietal network (JC 0.07). The networks that were the least involved in early $\mathrm{A} \beta$ accumulation were the sensorimotor (JC 0.01) and visual networks (JC 0.01).

Validation of our $\mathrm{A} \beta$ staging by identifying late $\mathrm{A} \beta$ regions. We have used the CSF+/PET- subjects as a proxy for early stage $\mathrm{A} \beta$ accumulators and compared them with CSF-/PET- subjects (non-accumulators) to identify early $\mathrm{A} \beta$ regions. If our CSF/PET groups are valid for staging $A \beta$ phases, we should also be able to compare early $\mathrm{A} \beta$ accumulators and $\mathrm{CSF}+\mathrm{PET}+$ subjects (our proxy for late stage $A \beta$ accumulators) to identify known regions of late $A \beta$ accumulation. The results from the ROI-based comparisons are shown in Table 3 and Supplementary Movie 2. The largest differences were seen in the precentral, postcentral, paracentral, pericalcarine, and lingual regions. This means that our proxy group for late $\mathrm{A} \beta$ accumulation $(\mathrm{CSF}+\mathrm{PET}+)$ had a higher accumulation rate of the $\mathrm{A} \beta$ PET signal over time compared with early $\mathrm{A} \beta$ accumulators (CSF+/PET-) around the sensorimotor cortex and occipital lobe. Note that these significant, late $A \beta$ regions did not overlap with the early $A \beta$ regions (compare Tables 2 and 3). The corresponding voxelwise 

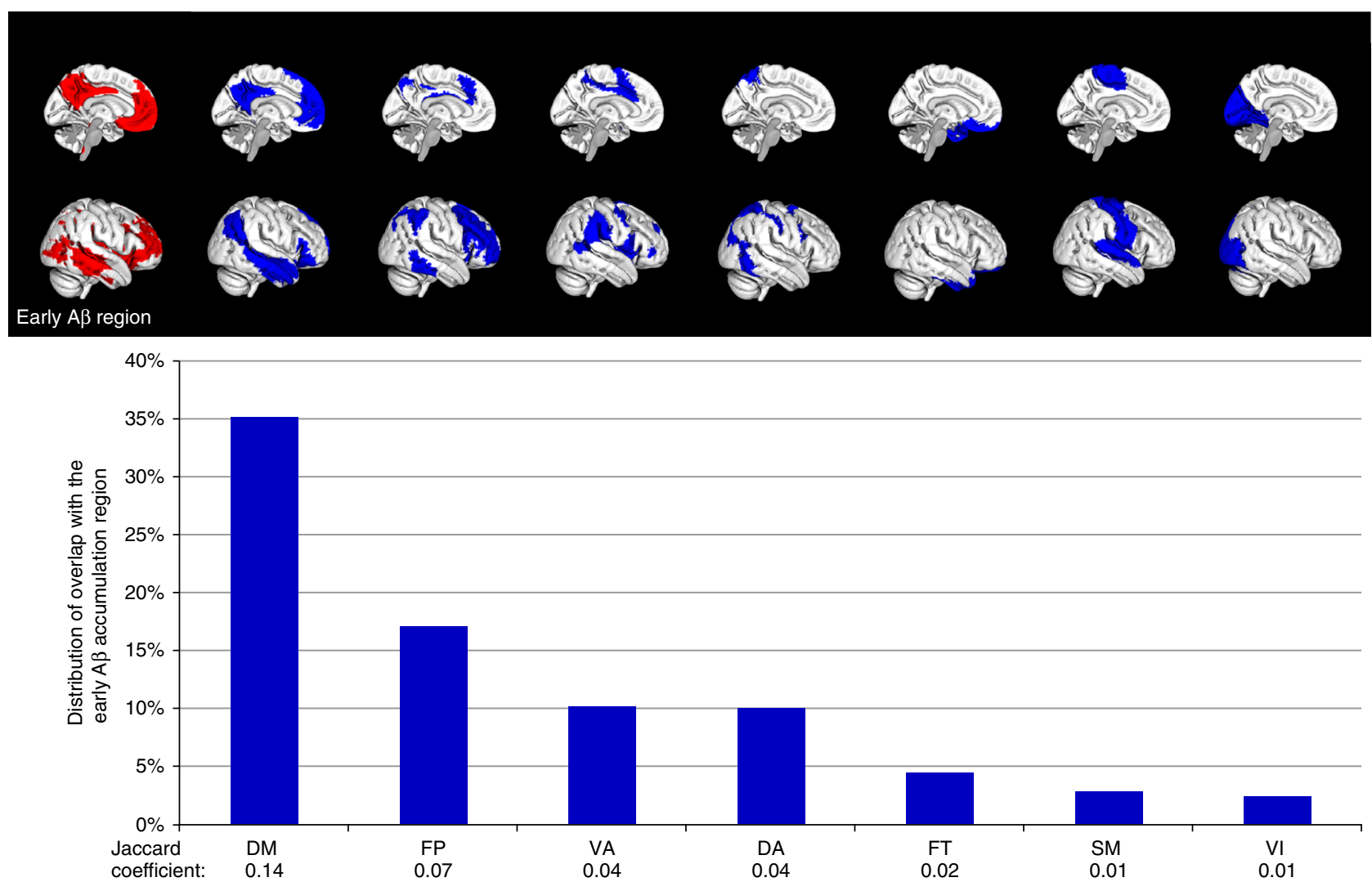

Fig. 2 Distribution of early $A \beta$ accumulation among functional networks in ADNI. The bars show the distribution of the overlap between the significant cluster of early $A \beta$ accumulation (shown in red, see also Fig. 1a) and the functional network ROls (shown in blue above the bars). The sum of the seven bars is therefore $100 \%$. The pattern of early stage A $\beta$ fibrils overlapped mostly with the DM followed by the FP network. The Jaccard coefficient describes the similarity between the early $A \beta$ accumulation region and the functional network ROIs. It is calculated as the overlap between the early $A \beta$ region and the network ROI/(early $\mathrm{A} \beta$ region + network ROI - overlap between early A $\beta$ region and the network ROI). DA dorsal attention, DM default mode, FP frontoparietal, FT frontotemporal, SM sensory motor, VA ventral attention, VI visual network

comparisons between early and late accumulators are shown in Fig. 3 and highlight similar late regions (greatest significant difference in the sensorimotor cortex and parts of the occipital lobe).

Atrophy and glucose metabolism in relation to $\mathrm{A} \beta$ accumulation. To test the hypothesis that atrophy, as a marker of neuronal and synaptic degeneration, starts after the initiation of $\mathrm{A} \beta$ accumulation, we compared the longitudinal change in gray matter (GM) volumes between the CSF/PET groups using voxel-based morphometry (VBM) analysis (Fig. 4a, b and Supplementary Fig. 2A). No clear atrophy pattern was seen in early $A \beta$ accumulators (CSF+/PET-) compared with non-accumulators (CSF-/PET-) (Fig. 4a). In contrast, late $\mathrm{A} \beta$ accumulators $(\mathrm{CSF}+/ \mathrm{PET}+)$ exhibited the expected longitudinal atrophy pattern of over time involving mainly temporoparietal regions when comparing them to non-accumulators (Fig. 4b) and early A $\beta$ accumulators (Supplementary Fig. 2A). Similar analyses were made with longitudinal FDG PET data to examine changes in glucose metabolism in relation to early $\mathrm{A} \beta$ accumulation. There were no differences in FDG uptake over time between nonaccumulators (CSF-/PET-) and early A $\beta$ accumulators (CSF +/PET-) (Fig. 4c), while a cortical metabolic reduction in the temporal lobe was seen in late $\mathrm{A} \beta$ accumulators $(\mathrm{CSF}+/ \mathrm{PET}+)$ compared with non-accumulators (CSF-/PET-) (Fig. 4d) and early $\mathrm{A} \beta$ accumulators (CSF+/PET-) (Supplementary Fig. 2B).

Replication of the early A $\beta$ regions in the BioFINDER cohort. To test the generalizability of our results we replicated the early
$\mathrm{A} \beta$ accumulation regions in the BioFINDER cohort. Only crosssectional data was available from the BioFINDER study. Although longitudinal comparisons are preferred given our hypothesis, regional differences in $\mathrm{A} \beta$ deposition should still be noticeable when comparing CSF+/PET- and CSF-/PET- subjects crosssectionally. A strength of this cohort is that another A $\beta$ PET ligand $\left({ }^{18} \mathrm{~F}\right.$-flutemetamol) as well as another immunoassay for CSF A $\beta$ (INNOTEST) were used, and the results would therefore be more generalizable if they were replicable in this cohort. The characteristics of BioFINDER is provided in Supplementary Table 2. Similar early A $\beta$ accumulation regions were identified as in ADNI when comparing cross-sectional ${ }^{18}$ F-flutemetamol SUVRs between CSF+/PET - and CSF-/PET - subjects using ROI-based analyses in BioFINDER (Supplementary Table 3). The most significant regions were found in the orbitofrontal cortex, the posterior cingulate cortex, and the precuneus (Supplementary Table 3, which can be compared with ADNI data in Table 2). Further, the cross-sectional voxelwise comparisons between CSF +/PET - and CSF-/PET- subjects in BioFINDER showed significant changes predominantly in the orbitofrontal cortex and posterior cingulate similar to the longitudinal analyses in ADNI (Fig. 5, compare with ADNI data in Fig. 1a, b).

Early $\mathbf{A} \beta$ accumulation and functional connectivity. Restingstate functional MRI was available in the BioFINDER study and this cohort was therefore used for this analysis. To examine the association between early $\mathrm{A} \beta$ accumulation and functional networks beyond the anatomical similarities (Fig. 2) we correlated 
Table 3 Annual $\mathbf{A} \beta$ accumulation rates in the significant late $\mathbf{A} \beta$ regions in $\mathbf{A D N I}$

\begin{tabular}{|c|c|c|c|}
\hline Region & CSF+/PET- & CSF+/PET+ & $p$-value \\
\hline Precentral, right & $0.1 \%(-0.5-0.7)$ & $2.1 \%(1.7-2.6)$ & 0.000002 \\
\hline Postcentral, right & $0.1 \%(-0.7-0.9)$ & $2.3 \%(1.8-2.7)$ & 0.000005 \\
\hline Pericalcarine, left & $0.2 \%(-0.8-1.3)$ & $2.8 \%(2.2-3.6)$ & 0.000006 \\
\hline Postcentral, left & $0.2 \%(-0.5-1.2)$ & $2.4 \%(1.9-2.8)$ & 0.000008 \\
\hline Precentral, left & $0.3 \%(-0.3-1.0)$ & $2.3 \%(1.9-2.9)$ & 0.000024 \\
\hline Paracentral, left & $0.6 \%(-0.2-1.4)$ & $2.4 \%(1.9-2.8)$ & 0.000057 \\
\hline Lingual, left & $0.5 \%(-0.7-1.9)$ & $2.8 \%(2.2-3.4)$ & 0.00019 \\
\hline Lateral occipital, left & $0.4 \%(-0.4-1.3)$ & $2.3 \%(1.8-2.9)$ & 0.00026 \\
\hline Pericalcarine, right & $0.4 \%(-0.6-1.5)$ & $2.9 \%(2.3-3.6)$ & 0.0003 \\
\hline Paracentral, right & $0.6 \%(-0.2-2.1)$ & $2.2 \%(1.7-2.7)$ & 0.00038 \\
\hline Fusiform, left & $1.1 \%(0.3-2.0)$ & $2.4 \%(2.0-2.8)$ & 0.00049 \\
\hline Lingual, right & $0.6 \%(-0.4-1.8)$ & $2.8 \%(2.2-3.5)$ & 0.0006 \\
\hline Cuneus, left & $-0.3 \%(-1.2-0.8))$ & $2.0 \%(1.4-2.8)$ & 0.00062 \\
\hline Middle temporal, left & $1.1 \%(0.3-2.0)$ & $2.2 \%(1.9-2.6)$ & 0.0007 \\
\hline Lateral occipital, right & $0.6 \%(-0.1-1.4)$ & $2.4 \%(1.9-3.1)$ & 0.00088 \\
\hline Fusiform, right & $0.9 \%(0.0-1.8)$ & $2.4 \%(1.9-2.9)$ & 0.0017 \\
\hline Inferior temporal, right & $1.3 \%(0.5-2.2)$ & $2.4 \%(1.9-2.8)$ & 0.0022 \\
\hline Inferior temporal, left & $1.5 \%(0.7-2.5)$ & $2.6 \%(2.2-3.1)$ & 0.003 \\
\hline Superior temporal sulcus, left & $1.4 \%(0.6-2.3)$ & $2.4 \%(1.9-2.8)$ & 0.0047 \\
\hline Caudal middle frontal, left & $1.3 \%(0.5-2.1)$ & $2.5 \%(2.0-3.1)$ & 0.0054 \\
\hline Superior parietal, left & $1.0 \%(0.2-1.9)$ & $1.9 \%(1.5-2.3)$ & 0.0074 \\
\hline Superior frontal, left & $1.2 \%(0.5-2.0)$ & $1.9 \%(1.5-2.3)$ & 0.013 \\
\hline
\end{tabular}

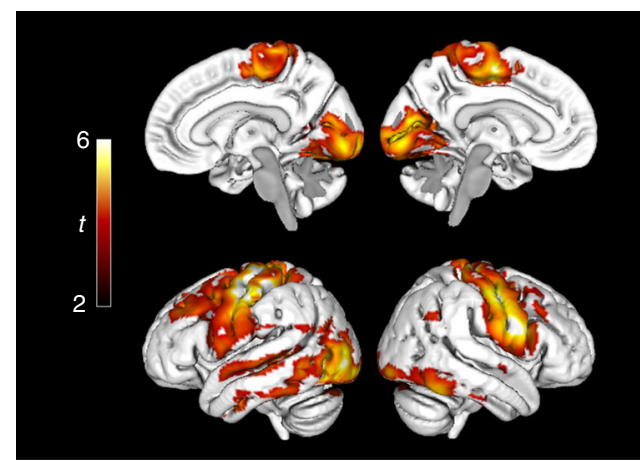

Fig. 3 Late $A \beta$ accumulation regions in ADNI. Voxelwise comparisons of annual SUVR change in early $A \beta$ accumulators (CSF+/PET-, $n=59$ ) and late $A \beta$ accumulators (CSF+/PET,$+ n=191)$. A significantly increased SUVR rate is seen in the sensorimotor cortex, occipital lobe, and dorsal temporal lobe in late compared with early $A \beta$ accumulators. Voxelwise two-sample $t$ tests were used and all comparisons were adjusted for age and gender. The significance threshold was set at $p<0.001$. The red and yellow colors illustrate significant $t$ values according to the scale on the left

levels of CSF A $\beta 42$ with whole-brain connectivity (Fig. 6). The groups of interest were the early $\mathrm{A} \beta$ accumulators (CSF+/PET-) and biomarker normal individuals with close to abnormal CSF $\mathrm{A} \beta 42\left(\mathrm{CSF}_{-\mathrm{low}} / \mathrm{PET}-\right)$. In the CSF+/PET- group we found a significant network component where reduced connectivity was associated with decreasing levels of CSF A 342 . The component predominantly consisted of intra-DMN links, but also links between the DMN and the frontoparietal network (Fig. 6a). The summed connectivity on the significant correlation component changed most drastically when CSF A $\beta 42$ levels were closer to the cut-off (400-516 ng/L for the INNOTEST ELISA) and seemed to reach a floor effect as A $\beta 42$ reached levels of around $350 \mathrm{ng} / \mathrm{L}$.
Figure $6 \mathrm{c}$ depicts the intra-DMN links, which dominate the significant hypoconnectivity component correlating with lower CSF $\mathrm{A} \beta 42$ values in the early $\mathrm{A} \beta$ accumulators. Furthermore, a significant network component involving the DMN was associated with CSF A $\beta 42$ in the $\mathrm{CSF}_{- \text {low }} / \mathrm{PET}$ - group, but with fewer constituent links (Fig. 6b). Interestingly, the correlation was in the opposite direction showing an increased connectivity as CSF A $\beta 42$ levels dropped from normal levels towards the abnormal cut-off. In summary, Fig. 6 indicates that when CSF A $\beta 42$ levels drop within the near abnormal range they might be associated with an increased connectivity in connections involving the DMN. Then, when CSF A $\beta 42$ levels become abnormal and decrease further as $\mathrm{A} \beta$ accumulates, they are instead clearly associated with a decreased connectivity within the DMN and mainly between the DMN and the frontoparietal network. The associations with hypoconnectivity and hyperconnectivity were similar when adjusting for CSF P-tau and T-tau, indicating the independent effect of $\mathrm{A} \beta$ on the connectivity (Supplementary Fig. 3).

\section{Discussion}

This study examined where $A \beta$ fibrils are more prone to start accumulating, by comparing the longitudinal outcome of nondemented subjects with early signs of $A \beta$ accumulation (CSF $+/ \mathrm{PET}-$ ) to those with no measurable signs of $\mathrm{A} \beta$ accumulation (CSF-/PET-), in a large cohort of 473 subjects. The results showed significantly increased rates of $\mathrm{A} \beta$ fibril accumulation predominantly in the precuneus, posterior cingulate cortex, and orbitofrontal cortex in this early $A \beta$ stage. When examining subjects with even earlier signs of $\mathrm{A} \beta$ accumulation (CSF-/PETsubjects who converted to CSF+/PET- within 2 years), significantly increased $A \beta$ fibril accumulation rate was again seen in the medial orbitofrontal and posterior cingulate cortex, compared with stable CSF-/PET- subjects. These regions were replicated in an independent cohort $(n=406)$. To our knowledge, this is the 


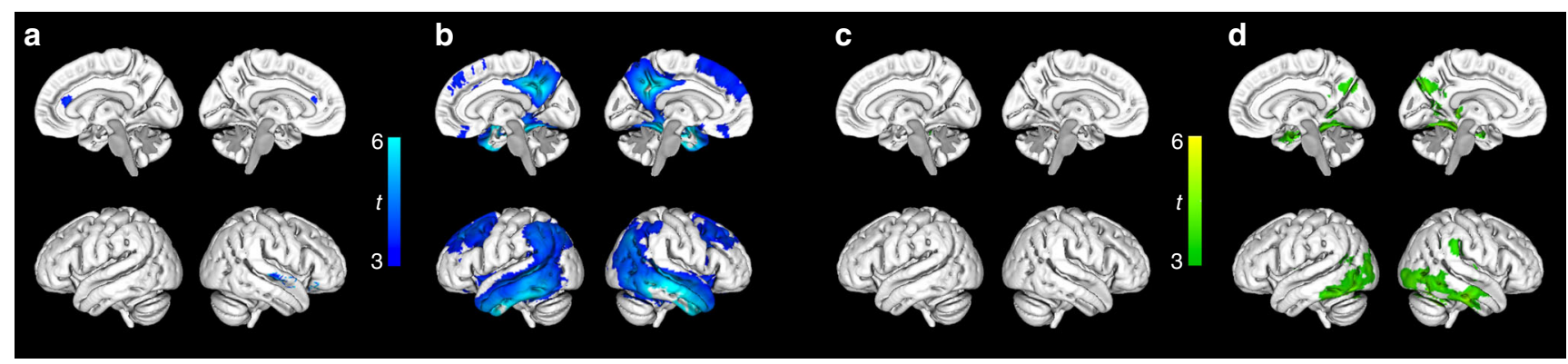

Fig. 4 Group comparisons of annual change in brain volume and glucose metabolism in ADNI. a Voxel-based morphometry (VBM) comparison of annual MRI change over 2 years in early A $\beta$ accumulators (CSF+/PET-, $n=59$ ) compared with non-accumulators (CSF-/PET-, $n=218$ ). The results are adjusted for age, gender, and intracranial volume. The contrast is reduction in cortical thickness in early compared with non-accumulators and shows no clear pattern of longitudinal atrophy in the early $A \beta$ accumulators ( CSF+/PET-). $\mathbf{b}$ The same VBM analysis as in $\mathbf{a}$, but with a comparison between nonaccumulators and late $A \beta$ accumulators (CSF+/PET+, $n=191)$. The contrast is reduction in late compared with non-accumulators and shows a typical $A D$ atrophy pattern in the non-demented late $A \beta$ accumulators (CSF+/PET+). c Voxelwise analysis of annual FDG PET change over 2 years in nonaccumulators $(n=153)$ compared with early A $\beta$ accumulators $(n=41)$. No significant difference is seen. $\mathbf{d}$ The same FDG PET analysis as above, but with a comparison between non-accumulators and late $A \beta$ accumulators $(n=124)$. The contrast is reduction in glucose metabolism in late compared with early $A \beta$ accumulators and shows a temporal and to a lesser extent parietal reduction in metabolism in the non-demented late $A \beta$ accumulators. Voxelwise twosample $t$-tests were used and all results in a-d were adjusted for age and gender. The VBM analysis was also adjusted for total intracranial volume. Only voxels with a $p<0.001$ are shown. The colors illustrate significant $t$ values according to the scales

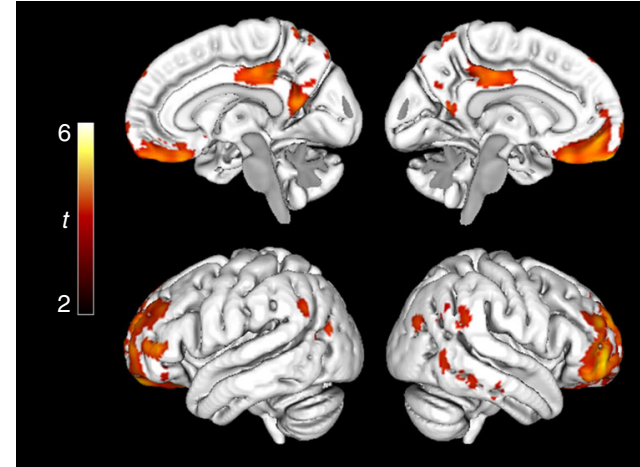

Fig. 5 Replication of the early A $\beta$ regions in BioFINDER. Comparison of ${ }^{18} \mathrm{~F}$ flutemetamol SUVR between early accumulators (CSF+/PET-, $n=30)$ and non-accumulators (CSF-/PET-,$n=219$ ) in BioFINDER to identify early $A \beta$ regions. The highest significance was seen around the posterior regions of the cingulate cortex and the orbitofrontal cortex similar to the results in ADNI (Fig. 1a, b). Note that the BioFINDER analysis included cross-sectional data and fewer subjects compared to ADNI, which results in less statistical power. Voxelwise two-sample $t$-test was used. The significance threshold was set at $p<0.001$ and the comparison was adjusted for age and gender. The red/yellow colors illustrate significant $t$ values according to the scale on the left

first study to identify a group of early stage $\mathrm{A} \beta$ accumulators and detect increased $A \beta$ accumulation rates specifically in these regions using longitudinal data. Previous longitudinal studies have either failed to detect an early increase in regional $A \beta$ deposition $^{16}$ or identified a rather large early stage $A \beta$ region including the posterior temporal, inferior parietal, posterior cingulate, precuneus, and occipital areas ${ }^{4}$. Those previous results indicate that there might have been methodological issues in correctly identifying the preclinical $\mathrm{AD}$ cases who have just started to accumulate $A \beta$.

There are a few previous studies where cross-sectional results have been used to approximate early stage $A \beta$ accumulation regions. In a subanalysis of a study aimed to identify different $A \beta$ PET cut-offs, voxelwise comparisons were made between a reference group with very low A $\beta$ PET SUVR $(n=22)$ and several groups of subjects ( $n=22$ in each group) with increasingly higher global SUVRs ${ }^{17}$. They found that the first significant SUVR differences between the controls and a group with sub-threshold SUVRs were located in the medial frontal cortex and the precuneus, similar to our early $A \beta$ regions. Another cross-sectional study used a different methodology to approximate the cortical spread of $A \beta$ in 67 healthy controls and 128 patients with MCI or AD dementia ${ }^{18}$. By assuming that earlier regions would show increased $A \beta$ PET signal in a greater number of subjects, they found an initial $A \beta$ region involving the areas in Fig. 1a, but also affecting middle and inferior temporal regions, and superior and middle frontal regions. In neuropathological studies, the results are mixed. One study reported that the presumably first $A \beta$ phase had $A \beta$ deposits throughout the neocortex ${ }^{5}$, while a prior publication by Braak \& Braak ${ }^{19}$ showed an early pattern of $A \beta$ deposits in the basal parts of the medial and anterior regions of the prefrontal cortex, and the basal anterior temporal lobe. The differences are probably partly explained by the different staining methods and their affinity to different $A \beta$ forms (discussed in the methodological aspects below). A clear advantage of the present study is that longitudinal $A \beta$ changes in the whole brain has been analyzed, instead of cross-sectional examinations in predetermined regions as in neuropathological studies.

The early $A \beta$ regions we identified were mainly located within the DMN (Fig. 2). The DMN has a core network located in the posterior cingulate cortex, precuneus, and medial prefrontal $\operatorname{cortex}^{20}$. It is almost always active, but increasingly so during daydreaming, activation of episodic memory and other internally directed activities and decreasingly during task-based activities and interaction with the external environment ${ }^{21,22}$. A certain overlap between the DMN and the distribution of $A \beta$ fibrils in $\mathrm{AD}$ has also been noted in previous publications $\mathrm{s}^{23-26}$. The cause of $A \beta$ aggregation within the DMN is currently unknown. One explanation is that the deposition of $\mathrm{A} \beta$ fibrils could be caused by the high neuronal activity within the DMN, which is supported by animal studies showing that $A \beta$ secretion and deposition is enhanced by neuronal activity ${ }^{27-29}$. An alternative explanation is that $A \beta$ accumulation is caused by the high metabolic demand and metabolic stress in these neurons (high energy consumption 
a

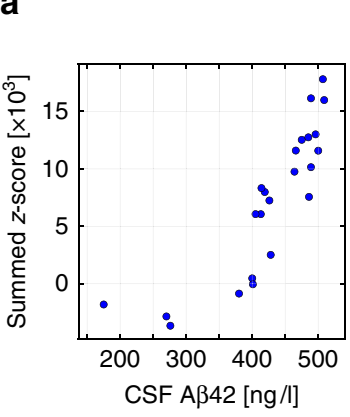

b

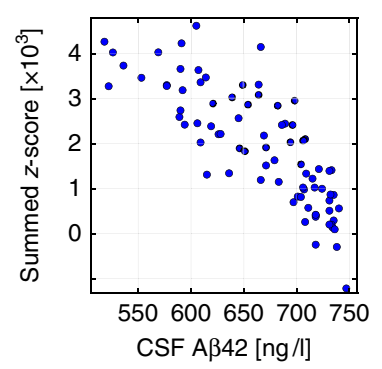

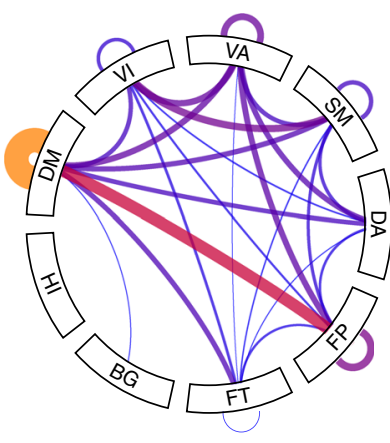

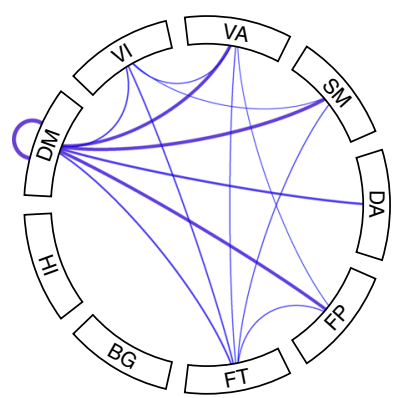

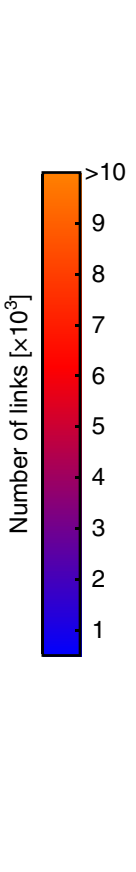

C

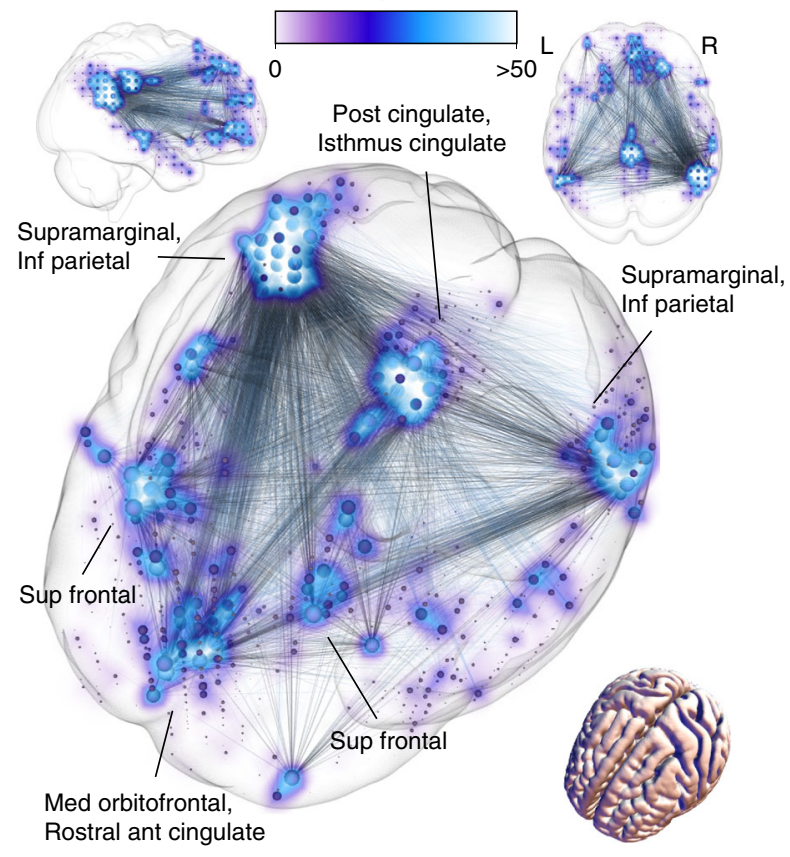

Fig. 6 Association between functional connectivity and early A $\beta$ accumulation in BioFINDER. $\mathbf{a}$ and $\mathbf{b}$ show CSF A $\beta 42$ correlations with whole-brain restingstate fMRI connectivity in non-demented subjects with normal A $\beta$ PET and QC-passed fMRI data $(n=103)$. Nodes were grouped into the 7 networks and 2 subcortical regions in the connectograms (a and $\mathbf{b}$ ). The connectograms show that the significant network component associated with CSF A 342 was qualitatively similar for the CSF+/PET- (a) and CSF-low/PET- (b) groups and consisted mostly of intra- and inter-DM connections. Intra-DM dominated and the strongest inter-DM connection was with the frontoparietal network. The network component of the CSF+/PET- group exhibited reduced connectivity with decreasing CSF A 42 (a), $r=0.91, p<0.001$. By contrast, in the qualitatively similar network component of the CSF-low/PET- group, decreasing $A \beta 42$ was instead associated with increased connectivity $(\mathbf{b}), r=-0.85, p<0.001$. Panel $\mathbf{c}$ shows the dominating intra DM-reduction in connectivity for the CSF+/PET- group (a). In summary, a show that A $\beta$ accumulation measured with CSF A $\beta 42$ in early A $\beta$ accumulators is associated with hypoconnectivity in intra- and inter-DM network links. $\mathbf{b}$ instead shows that in a population including subjects with even earlier indications of $A \beta$ accumulation (CSF-low/PET-) A $\beta$ accumulation is instead associated with hyperconnectivity in similar neuronal connections. Correlation coefficients ( $r$ ) refer to Spearman correlation between summed connectivity and CSF A $\beta 42$ levels. Age, gender, and APOE $\varepsilon 4$ status was controlled for by partial correlation. Network components correlating with CSF A 442 were calculated using a method similar to the NBS algorithm (see "Methods for the BioFINDER study" for statistical details). Acronyms: BG basal ganglia, CSF-low normal CSF A 342 levels close to the abnormal cut-off (517-750 ng/L), DA dorsal attention, DM default mode, FP frontoparietal, FT frontotemporal, HI hippocampus, QC quality control, SM sensorimotor, VA ventral attention, VI visual network

and frequent fluctuations in activation and deactivation) and not the neuronal activation per $\mathrm{se}^{30}$. Although the core DMN was the most prominent network that we found that had anatomical overlap with the early $A \beta$ regions, we want to point out that involvement of other networks was also evident in the early $\mathrm{A} \beta$ accumulation, especially the frontoparietal network (Fig. 2). This indicates that early stage accumulation is not related to a unique feature of the $\mathrm{DMN}$, but in a more general sense to hubs in the brain with high connectivity. This interpretation is supported by previous studies showing an association between $\mathrm{A} \beta$ distribution and several networks and network $\operatorname{hubs}^{25}, 31,32$.

The association between functional networks and earliest stages of $\mathrm{A} \beta$ accumulation was further examined using functional connectivity analyses in BioFINDER. When we correlated wholebrain functional connectivity and CSF A $\beta 42$ levels in the early stage $\mathrm{A} \beta$ accumulators (CSF+/PET-), we found a decreased connectivity in intra-DMN connections and between DMN and the frontoparietal network as CSF A $\beta 42$ decreased (Fig. 6a). The disruption of these connections in BioFINDER is especially interesting given the distribution of early $A \beta$ fibrils in ADNI in the DMN and frontoparietal networks (Fig. 2). This correlation between the disruption of functional connectivity and $\mathrm{A} \beta$ pathology has recently been described in $\mathrm{AD}^{32}$, 33, but to our knowledge the present study is the first to show that this association is present already at the very earliest preclinical stages of $\mathrm{AD}$ when $\mathrm{A} \beta$ fibrils are just starting to accumulate. In our second connectivity analysis (Fig. 6b) we examined the correlation between functional connectivity and CSF A $\beta 42$ in those with indications of very early $\mathrm{A} \beta$ accumulation (close to abnormal CSF $\mathrm{A} \beta 42$ and normal A $\beta$ PET; $\mathrm{CSF}_{-\mathrm{low}} / \mathrm{PET}-$ ). The results indicate that when $A \beta 42$ drops towards abnormal levels in the CSF the connectivity instead increases in similar DMN-related connections in which there was a positive correlation between decreased connectivity and decreasing levels of CSF A 342 within the abnormal A $\beta 42$ range (compare connectograms in Fig. 4a, b). Interpretations of this preliminary finding are speculative, but it might be that cortical hubs like the medial frontal lobe and posterior cingulate exhibit increased activity before $A \beta$ fibrilcontaining senile plaques are formed in preclinical AD. This is supported by experimental cell and animal work suggesting that neural activity leads to increased $A \beta$ generation and secretion $^{27,34}$. Later, the hypoconnectivity might develop when $\mathrm{A} \beta$ fibrils starts accumulating and causes neuronal dysfunction. Alternatively, early and soluble $\mathrm{A} \beta$ oligomers might instead trigger the initial hyperactivity, which is supported by studies in 
transgenic $\mathrm{AD}$ models suggesting that neurons become hyperactive very early, independently of the deposition of $A \beta$ into plaques, and that the silencing of neurons emerges only later in the disease course ${ }^{35}$.

Although altered functional connectivity was found already in the early $\mathrm{A} \beta$ accumulators ( $\mathrm{CSF}+\mathrm{PET}-$ ), our longitudinal analyses (Fig. $4 \mathrm{a}, \mathrm{c}$ ) showed that no changes in glucose metabolism or atrophy were present at this stage. However, decreased temporoparietal glucose metabolism and atrophy was observed in the late $\mathrm{A} \beta$ accumulators (CSF+/PET+; Fig. $4 \mathrm{~b}$, $\mathrm{d}$ and Supplementary Fig. 2A, B). This finding can have practical implications for the enrollment in anti-A $\beta$ clinical trials since anti-A $\beta$ agents should be introduced after $A \beta$ accumulation has started but before neurodegeneration is present. This should make CSF+/PETsubjects suitable for inclusion in clinical trials, especially if the drug is intended to target $A \beta$ accumulation over long time periods using $\mathrm{AD}$ biomarkers as outcomes of its efficacy in this preclinical disease stage.

The temporal sequence of $\mathrm{A} \beta$ accumulation preceding neurodegeneration confirms the most acknowledged hypothetical model on the development of $\mathrm{AD}^{1}$. It also strengthens the rationale for using CSF/PET groups as proxies for $A \beta$ stages in $\mathrm{AD}$. To further validate the use of CSF/PET groups, we compared $\mathrm{A} \beta$ accumulation in our proxy group for early $A \beta$ accumulation $(\mathrm{CSF}+/ \mathrm{PET}-)$ with the proxy group for late $\mathrm{A} \beta$ accumulation $(\mathrm{CSF}+/ \mathrm{PET}+)$. As hypothesized, we identified known regions of late stage $\mathrm{A} \beta$ accumulation, including the sensorimotor cortex and occipital lobe (Fig. 3, Supplementary Movie 2 and Table 3$)^{3,19}$. In a recent study, we also showed that CSF+/PETsubjects accumulated $\mathrm{A} \beta$ at a similar rate as $\mathrm{CSF}+/ \mathrm{PET}+$ subjects and about four times higher than CSF-/PET- subjects ${ }^{12}$. The high accumulation rate in $\mathrm{CSF}+\mathrm{PET}$ - subjects was not accompanied by cognitive decline, but we found a subtle decline in memory over 5 years in $\mathrm{CSF}+\mathrm{PET}+$ subjects that further validates the use of CSF/PET groups for stratifying $\mathrm{A} \beta$ stages. Nonetheless, we acknowledge that CSF+/PET- subjects are not all early $\mathrm{A} \beta$ accumulators since this state also can be caused by CSF analytical factors, failed PET scans, and medical conditions other than $\mathrm{AD}^{36-38}$.

As part of our methodology we chose to primarily compare longitudinal changes between the CSF/PET groups instead of comparing cross-sectional values or just within-group changes to the identify the early $A \beta$ regions. This had the advantage of accounting for physiological differences in $\mathrm{A} \beta$ burden, age-related non-specific $A \beta$ accumulation rates, and the fact that the individuals within each CSF/PET stage had reached slightly different time points in the disease progression. In addition, the early $\mathrm{A} \beta$ accumulating brain regions remained significant when adjusting for confounding factors such as longitudinal changes in cortical thickness, $A P O E$ genotype and clinical status. These adjustments show that the results were not confounded by differences in atrophy between the groups or differences between healthy controls and MCI subjects (discussed further in the Methods section). It should be noted that the early $A \beta$ regions are derived from group analyses, which show where $A \beta$ is prone to start accumulating but they do not exclude the possibility that the accumulation might start in other regions in some individuals.

A limitation of our early $A \beta$ regions is that they have been identified using PET ligands that bind to primarily dense-core $A \beta$ aggregates and to a lesser extent or not at all to diffuse plaques $^{39,40}$. We can therefore not pinpoint the exact $A \beta$ forms within the early $A \beta$ regions, neither can we say if $A \beta$ aggregates not detected by PET are present in other areas of the brain at this early stage. Future PET ligands that binds to different forms, e.g., $\mathrm{A} \beta$ protofibrils, might reveal a different early $\mathrm{A} \beta$ pattern ${ }^{41}$. The binding affinity of current PET ligands could explain some of the differences with neuropathological studies that either use silver technique ${ }^{19}$, which stain not only $\mathrm{A} \beta$ but also tau, or anti-A $\beta$ antibodies $^{5}$ that bind to specific $\mathrm{A} \beta$ epitopes and stain different $\mathrm{A} \beta$ forms. Nonetheless, a strength of the methodology is that we could detect similar early $A \beta$ regions in two different cohorts using two different types of PET ligands; ${ }^{18} \mathrm{~F}$-florbetapir (a stilbene compound) and ${ }^{18} \mathrm{~F}$-flutemetamol (an ${ }^{18} \mathrm{~F}$-labeled analog of ${ }^{11} \mathrm{C}$-Pittsburgh Compound $\mathrm{B}(\mathrm{PiB})$ derived from thioflavin $\left.\mathrm{T}\right){ }^{40}$ The cut-offs for the two PET ligands were established separately in each cohort, and the data were not pooled in the analyses, nor were the groups compared to each other. We therefore chose to not transform SUVR into a standardized scale such as centiloid units $^{42}$.

To conclude, we believe that the identified early $\mathrm{A} \beta$ regions can have several important implications. These brain regions give a pathophysiological insight into where $A \beta$ fibril accumulation is prone to start in $\mathrm{AD}$ and its relationship to functional networks and their connectivity. Our data indicated that $\mathrm{A} \beta$ starts accumulating before overt metabolic changes or atrophy, and that hypoconnectivity within the early $A \beta$-accumulating regions has already occurred when $A \beta$ fibrils just starts to accumulate. The most prominent early $A \beta$-accumulating regions were the precuneus, posterior cingulate cortex, and the medial orbitofrontal cortex, possibly with an overlap to the anterior cingulate. Based on our results, future studies should be able to examine early unique pathophysiological events and triggering mechanisms of $\mathrm{A} \beta$ accumulation by comparing molecular and physiological properties of the early-accumulating regions to lateraccumulating regions. These early-accumulating $\mathrm{A} \beta$ regions should also be advantageous to use in an A $\beta$ PET composite ROI to better assess early fibrillar $\mathrm{A} \beta$ deposition (available at http:// biofinder.se), which can have practical implications for early $\mathrm{AD}$ diagnostics, $A \beta$ staging and enrollment in clinical trials that target $\mathrm{A} \beta$ accumulation.

\section{Methods}

Study data. Longitudinal data from the ADNI were used for all the statistical analyses in the study, except for replicating the finding of early $\mathrm{A} \beta$ accumulating regions and for the functional connectivity analyses for which data from the Swedish BioFINDER study were used. The methodology described in the next sections refers only to analyses performed in the ADNI cohort. Methodology referring to analyses in the BioFINDER cohort is described separately under Methods for the BioFINDER study.

Participants. Only non-demented individuals were included in this study (characterized as either cognitively normal or diagnosed with mild cognitive impair ment, MCI). Demented subjects were excluded since the study aim was to identify early $\mathrm{AD}$ pathology. We chose to include MCI subjects in addition to healthy controls since the cognitive impairment did not necessarily have to be caused by an underlying $\mathrm{AD}$ process and because about a third of MCI subjects have shown to be incorrectly diagnosed with cognitive impairment ${ }^{43-45}$. We also attempted to include a range of different pre-dementia $\mathrm{AD}$ phases for a better staging of late $\mathrm{A} \beta$ accumulators, early $A \beta$ accumulators and non-accumulators. To account for this inclusion the models were adjusted for clinical status (MCI/healthy control) in subanalyses. The specific inclusion/exclusion criteria for the ADNI cohort can be found at http://www.adni-info.org. Briefly, all subjects were enrolled from the ADNI-2 study, were between the ages of 55 and 90 years, were fluent in Spanish or English, had completed at least six years of education, had a Mini-Mental State Examination score (MMSE) of $\geq 24^{46}$, and were free of any significant neurologic disease other than AD. Subjects classified as cognitively normal $(n=176)$ had a Clinical Dementia Rating scale $(\mathrm{CDR})$ score of $0^{47}$. MCI $(n=297)$ was defined as having preserved activities of daily living, absence of dementia, and an objective cognitive impairment as shown on the delayed recall test of the Wechsler Memory Scale - Logical Memory II as well as a CDR score of 0.5. Only subjects with a complete set of baseline and follow-up A $\beta$ PET scans and baseline CSF A $\beta 42$ data were included. Our study baseline was defined as the first visit where both CSF and PET data were available. Study subjects gave written informed consent and the study was approved by each participating site's Institutional Review Board.

Image acquisition. For MRI, 3 Tesla scanners were used. High-resolution 3D T1weighted images were acquired for volumetric measures, anatomical segmentation, 
and template normalization using an MPRAGE sequence (for details, see ref. ${ }^{48}$ ). Fibrillar $\mathrm{A} \beta$ pathology was measured using the PET ligand ${ }^{18} \mathrm{~F}$-florbetapir. Image data were acquired 50-70 minutes post-injection (see http://adni.loni.usc.edu/wpcontent/uploads/2010/05/ADNI2_PET_Tech_Manual_0142011.pdf). Glucose metabolism was measured employing FDG PET with images acquired 30-60 min post-injection. Florbetapir PET, FDG PET, and MR images from baseline and the 2-year follow-up visit were downloaded from the LONI database (http://ida.loni. usc.edu) and further processed locally. Longitudinal ${ }^{18} \mathrm{~F}$-florbetapir was available in all subjects, longitudinal MRI in 468 subjects (218 CSF-/PET-, 59 CSF+/PET-, and $191 \mathrm{CSF}+\mathrm{PET}+$ ), and longitudinal FDG PET in 318 subjects (153 CSF-/PET-, $41 \mathrm{CSF}+$ /PET-, and $124 \mathrm{CSF}+\mathrm{PET}+$ ).

Image processing. The motion corrected PET data was time-averaged and rigidly aligned with the anatomical MRI scan. SUVR were then calculated by normalizing to averages of appropriate reference regions. For florbetapir, the reference region consisted of the whole cerebellum, the pons/brainstem region, and eroded cortical white matter as this composite region has produced more reliable results in longitudinal analyses ${ }^{49}$. For FDG, a composite region comprising vermis/cerebellum superior to the horizontal fissure and pons were used as reference region ${ }^{50}$. Freesurfer version 5.3 (http://surfer.nmr.mgh.harvard.edu; Desikan-Killiany Atlas) segmentations of the structural MR images were applied to extract SUVRs from 68 predefined ROIs. Partial volume error (PVE) correction was performed for florbetapir SUVRs with the geometric transfer matrix (GTM) method ${ }^{51}$ using Freesurfer segmented regions ${ }^{52}$ (all florbetapir results shown in the tables and figures are from PVE corrected data).

The MRI volumes were further normalized to MNI152 standard space ${ }^{53}$ using a nonlinear diffeomorphic transformation ${ }^{54}$ enabling motion and PVE voxelwise ${ }^{55}$ corrected SUVRs to be transformed to MNI standard space for voxelwise analyses. VBM as implemented in Statistical Parametric Mapping (SPM) version 12 (Wellcome Department of Cognitive Neurology, London, UK; http://www.filion. ucl.ac.uk/spm) was used to evaluate GM intensity as a measure of atrophy. All individual T1-weighted MR images were segmented into tissue classes using SPM's unified segmentation algorithm. The GM segmentations were subsequently warped into a common MNI152 standard space using a cohort-specific template and the SPM Diffeomorphic Anatomical Registration Through Exponentiated Lie Algebra (DARTEL) toolbox and Jacobian scaled to estimate GM intensity. The resulting maps were smoothed with $8 \mathrm{~mm}$ full-width at half maximum (FWHM) Gaussian kernel. Intracranial volume (ICV) was calculated by summating the volumes of GM, white matter, and CSF based on the SPM tissue segmentations. Using the corrected SUVRs in standardized space, we also proceeded to calculate summed values within the functional MRI (fMRI) resting-state networks enumerated in the low resolution atlases with seven networks calculated by Yeo et al. (see details below) $)^{15}$.

Florbetapir and FDG PET images were co-registered to their respective MRI and subsequently warped into a common MNI152 space using the transformation information derived from warping the MRI scans. To assess changes over time in GM intensity (MRI), amyloid accumulation (florbetapir PET), and glucose metabolism (FDG PET) between baseline and follow-up examinations, parametric change maps were created. For longitudinal VBM, a midpoint average MRI was created for each subject based on the baseline and follow-up images using SPM12, resulting in Jacobian determinant maps showing local volumetric change between baseline and follow-up for each subject. Midpoint average images were GMsegmented and subsequently used to create a DARTEL template that was affinetransformed to the common MNI152 space. Midpoint average GM segmentations were multiplied by the Jacobian maps to create individual GM change maps, which were warped to the MNI-transformed DARTEL template and smoothed by an $8 \mathrm{~mm}$ FWHM isotropic Gaussian kernel to create longitudinal change maps. A GM mask was created using the same approach as for the cross-sectional data (see above). The florbetapir and FDG change maps were created using the warped images and FSL (http://fsl.fmrib.ox.ac.uk) according to the equation

$\mathrm{SUVR}_{\text {change }}=\left(\mathrm{PET} \mathrm{image}_{\text {followup }}-\right.$ PETimage $\left._{\text {baseline }}\right) /$ years between scans

CSF biomarkers. CSF A $\beta 42$, total tau (T-tau), and tau phosphorylated at Thr181 (P-tau) were measured using the multiplex xMAP Luminex platform (Luminex Corp, Austin, TX, USA) with the INNOBIA AlzBio3 kit (Innogenetics, Ghent, Belgium $)^{56,57}$. Data was combined from the available online files "UPENNBIOMK8.csv", "UPENNBIOMK7.csv, "UPENNBIOMK6_07_02_13.csv", and "UPENNBIOMK5_10_31_13.csv". Only cross-sectional CSF data from baseline were used in the analyses $(n=473)$, except for the analysis of "CSF converters" where longitudinal 2 -year data was used $(n=112)$. In the case of one subject with stable A $\beta 42$ levels, only 3-year follow-up data was available. The mean difference between baseline CSF sampling and the florbetapir PET scan was 9.6 days (ranging from 0 to 110 days).

Group classifications. All subjects were categorized into different groups according to their CSF A $\beta 42$ and A $\beta$ PET status. The following groups were derived from the baseline results: (1) normal CSF A $\beta 42$ and A $\beta$ PET (CSF-/PET-) referred to as non-accumulators $(n=218),(2)$ abnormal CSF A $\beta 42$ and normal A $\beta$ PET (CSF+/PET-) referred to as early A $\beta$ accumulators $(n=59)$, and (3) abnormal
CSF $\mathrm{A} \beta 42$ and $\mathrm{A} \beta$ PET (CSF+/PET + ) referred to as late $\mathrm{A} \beta$ accumulators $(n=191)$. Those with normal CSF A $\beta 42$ and abnormal A $\beta$ PET were not used in any analysis $(n=5)$. Abnormal CSF A 442 values were defined using the previously established cut-off of $<192 \mathrm{ng} / \mathrm{L}^{57-59}$. The cut-off for abnormal A $\beta$ PET SUVR was determined using a mixture modeling analysis since no previous cut-off had been established for PVE corrected florbetapir data in ADNI (using the GTM method). Mixture modeling statistics provide an unbiased threshold for abnormality (i.e., the diagnosis or status is not used in analysis) and it is a well-established method for defining $A \beta$ PET cut-offs ${ }^{12,17,60,61}$. To achieve a robust threshold we derived the cut-off from a larger ADNI sample $(n=663$, all non-demented subjects with CSF and A $\beta$ PET data). This resulted in a cut-off of $>0.872$ SUVR for an abnormal A $\beta$ PET using PVE correction and the composite reference region.

Methods for the BiofINDER study. The Swedish BioFINDER study was used for replicating the main results from ADNI and for the functional connectivity analyses. BioFINDER is a prospective study that focuses on identifying key mechanisms and improvement of diagnostics in $\mathrm{AD}$ and other neurodegenerative disorders. For details about study design, methods, and specific inclusion/exclusion criteria, see http://biofinder.se. The study was approved by the ethical review board in Lund, Sweden, and all participants gave their written informed consent. All nondemented individuals with CSF and A $\beta$ PET data were selected for this study. This resulted in a cohort consisting of cognitively healthy elderly subjects $(n=138)$ and consecutively recruited patients who had been referred to memory clinics due to cognitive complaints $(n=268)$. The PET ligand ${ }^{18} \mathrm{~F}$-flutemetamol was used for A $\beta$ PET, and images were acquired $90-110$ min post-injection. The PET scanning procedures have been described previously ${ }^{60}$. CSF A $\beta 42$ and P-tau were analyzed with INNOTEST ELISAs (Fujirebio Europe, Ghent, Belgium) and T-tau with EUROIMMUN ELISAs (EUROIMMUN AG, Lübeck, Germany) as previously described $^{60,62}$. Mixture modeling was performed in the sample to determine the cut-offs for abnormal CSF A $\beta 42(<517 \mathrm{ng} / \mathrm{L})$ and abnormal A $\beta$ PET $(>0.759$ SUVR). Only baseline data was available for analysis in the BioFINDER cohort.

Imaging was performed on a 3 Tesla Siemens Tim Trio scanner (Siemens Medical Solutions, Erlangen, Germany). The high-resolution 3D T1-weighted volume used for segmentation and normalization was acquired using an MPRAGE sequence (in-plane resolution $=1 \times 1 \mathrm{~mm}^{2}$, slice thickness $=1.2 \mathrm{~mm}$, TR $=$ $1950 \mathrm{~ms}, \mathrm{TE}=3.37 \mathrm{~ms}$, flip-angle $\left.=9^{\circ}\right)$. Spontaneous BOLD oscillations in the absence of external stimuli were imaged with a gradient-echo planar sequence (eyes closed, in-plane resolution $=3 \times 3 \mathrm{~mm}^{2}$, slice thickness $=3 \mathrm{~mm}, \mathrm{TR}=2000 \mathrm{~ms}$, $\mathrm{TE}=30 \mathrm{~ms}$, flip-angle $=90^{\circ}, 180$ dynamic scans, $6 \mathrm{~min}$ ).

Resting-state data preprocessing was performed with a pipeline composed of $\mathrm{FSL}^{63}, \mathrm{AFNI}^{64}$, and $\mathrm{ANTS}^{65}$. Anatomical processing involved skull stripping, segmentation of white matter (WM)/GM/CSF and normalization to MNI152space $^{53}$. Dropping the first five frames in anticipation of steady state, functional data was bulk motion, and slice timing corrected, furthermore nuisance regressed using the WM/CSF average signal, 6 components of physiological noise ${ }^{66}, 24$ motion parameters ${ }^{67}$, and linear/quadratic trends. Finally, the functional data was transformed to MNI space. Frames causing outliers in total frame-to-frame signal variation were censored, on average constituting $4 \%$ of the fMRI series ${ }^{68}$. The signal was band-pass filtered to $0.01-0.1 \mathrm{~Hz}$, further discriminating against scanner drift and physiological noise. No spatial smoothing was applied.

Subjects with a mean/maximum frame-wise displacement ${ }^{29}$, 69 exceeding 0.6/ $3.0 \mathrm{~mm}$ were excluded. As an extra precautionary step, the voxel-to-voxel BOLDsignal correlations across the whole brain (including GM, WM, and CSF) was calculated and summed. Outliers in this measure likely originate in a motioninduced global signal confound capable of eluding conventional motion estimation ${ }^{70}$ and were removed.

The processed fMRI data was resampled using trilinear interpolation to $6 \times 6 \times$ $6 \mathrm{~mm}^{3}$ resolution and masked with GM derived from a cortical resting-state network atlas ${ }^{71}$ and Harvard-Oxford subcortical atlas ${ }^{72}$. Fisher-z transformed Pearson correlation between the resulting $5071 \mathrm{GM}$ voxel time series then yielded a measure of functional connectivity (FC), corresponding to a weighted graph with nodes (voxels) and links (voxel BOLD time series correlations).

Network components correlating with CSF A $\beta 42$ were calculated using a method similar to the NBS algorithm ${ }^{73}$. We calculated the largest network component $C$ (defined as a connected set of links), for which $r_{i j}>r_{0}$, where $r_{i j}$ is the Spearman correlation over all subjects between CSF A $\beta 42$ and $z_{i j}$ for the link between voxel $i$ and $j$ (Fisher z-transformed voxel BOLD time-series correlation), and $r_{0}$ controls the component size and significance level of constituent links. We chose $r_{0}$ corresponding to approximately $p=0.001$ given the number of subjects in the calculation. Component size was then defined as the sum of all $r_{i j} \in C$ multiplied by the Spearman correlation of the sum of all $z$-values in the component and CSF levels. This last step allows for reasonable and natural variations on the extracted component. The component size was compared to a permutation-generated null distribution of sizes, thus controlling for the family-wise error rate in the weak sense at $\alpha=0.05$ The result of the algorithm is a network component on which sum of $z$-scores correlates significantly higher than for randomized sets of subject FC-CSF pairs. Age, gender and APOE $\varepsilon 4$ status was controlled for by partial correlation.

In order to simplify the analysis of network components, we grouped nodes using a resting-state network atlas ${ }^{71}$ containing: default mode, dorsal and ventral 
attention, sensory motor, visual, fronto-parietal, and fronto-temporal (medial temporal lobe/orbitofrontal cortex). To this set of labels we added two anatomically defined subcortical structures from the Harvard-Oxford atlas ${ }^{72}$ : the Basal Ganglia (BG: thalamus, caudate, putamen and pallidum) and hippocampus/amygdala (HI). Note that the permutation-based approach generates $p$ values for the network component as a whole, but since these are too large and complex to visualize, a network-based break up is necessary.

Only A $\beta$ PET negative subjects were used in the connectivity analysis. In addition to the previously described group of early $\mathrm{A} \beta$ accumulators (CSF+/PET-, $n=23$ after fMRI quality control) we also defined a group of biomarker negative subjects with indications of very early $\mathrm{A} \beta$ accumulation $(n=80)$. Those with low levels of CSF A $\beta 42$, but still within the normal range, have a high risk of becoming abnormal within the next couple of years ${ }^{13}$, which suggests that sub-threshold CSF $A \beta 42$ levels indicate very early $A \beta$ accumulation. This group was characterized as $\mathrm{A} \beta$ PET negative with CSF A $\beta 42$ between $517-750 \mu \mathrm{g} / \mathrm{mL}\left(\mathrm{CSF}^{-}{ }_{\text {low }} / \mathrm{PET}-\right.$ ).

Statistical analysis. Group comparisons of baseline characteristics (Table 1 and Supplementary Table 2) was performed with Mann-Whitney $U$ statistics if significant after the Kruskal-Wallis test. For ROI analyses, full factorial general linear models were used to analyze group differences and correlations of the 68 available cortical Freesurfer ROIs from the Desikan-Killiany Atlas. The dependent variable was $A \beta$ accumulation rate ([SUVR at follow-up - SUVR at baseline]/years between baseline and follow-up) in ADNI and baseline A $\beta$ PET SUVR in BioFINDER. The variance of the SUVRs were similar in each group. All results were adjusted for gender and age and, in the longitudinal analyses, time between A $\beta$ PET examinations. We also performed additional analyses adjusting for changes in cortical thickness over 2 years in each ROI and clinical status (healthy control/MCI) in addition to the above covariates. Longitudinal changes within a specific group of subjects were analyzed with the Paired T-test. Because of the limited number of subjects in the subanalysis of "CSF converters" $(n=11)$, we primarily used nonparametric statistics for this subgroup (the Mann-Whitney $U$ and Wilcoxon signed rank test). To control for multiple testing in the ROI-based analyses we used the Benjamini-Hochberg procedure with a conservative false discovery rate (FDR) of 0.05 (not to be confused with a $p$ value of 0.05$)^{74}$. Original $p$ values are presented for the ROI-based analyses but are only reported as significant if they are significant after the FDR correction. The effect size of the significant results when comparing the CSF/PET groups are given in \% annual increase of SUVR (yearly SUVR change divided by SUVR at baseline) for an easier comparison between different PET ligands and brain regions (and when comparing the results to other studies that use different reference regions). However, statistical significances were determined using actual SUVR or SUVR/year data. SPM12 was used for all voxelwise analyses. We employed voxelwise two-sample $t$-test and multiple regressions using age, gender, and ICV (for analyses involving VBM data) as covariates. All images had been transformed into common MNI space by using transformation measures from warping the co-registered MRI scans to the $2 \mathrm{~mm}$ MNI152 MRI template and smoothed with an $8 \mathrm{~mm}$ FWHM kernel. The resulting t-maps were thresholded to present results with a $p<0.001$ height, uncorrected for family-wise error, and a $k>100$ voxels cluster extent. For the resting-state correlation analysis, a component (consisting a connected set of links correlating with CSF biomarker levels above a $p=0.001$ threshold) was assigned a $p$ value as a whole based on the component size relative a permutation-generated null distribution, thus correcting for family-wise cerror in the weak sense (see "Methods for the BioFINDER study" for details). Finite mixture models for establishing biomarker cut-offs were performed with the package "mixtools" in R version 3.2.2 (R Foundation for Statistical Computing, Vienna, Austria, 2013). FDR correction of multiple comparisons was performed using the Benjamini-Hochberg formula in an Excel sheet (Microsoft Excel for Mac, 2011, version 14.4). All other statistical analyses were performed with SPSS for Mac, version 22.0 (SPSS Inc., Chicago, IL).

Data availability. MRI and PET images were downloaded online at https://ida. loni.usc.edu and further processed locally (see Image Processing above). All other ADNI data were also downloaded from the same site. BioFINDER data and processed ADNI data are not publicly available for download, but might be retrieved from the principal investigator Oskar Hansson. A PET-data-derived ROI of the early-accumulating $A \beta$ regions (Fig. 1a) is available in MNI152 space and Niftiformat at http://biofinder.se.

Received: 3 January 2017 Accepted: 23 August 2017

Published online: 31 October 2017

\section{References}

1. Jack, C. R. Jr. et al. Tracking pathophysiological processes in Alzheimer's disease: an updated hypothetical model of dynamic biomarkers. Lancet Neurol. 12, 207-216, (2013).
2. Sperling, R., Mormino, E. \& Johnson, K. The evolution of preclinical Alzheimer's disease: implications for prevention trials. Neuron 84, 608-622 (2014).

3. Suva, D. et al. Primary motor cortex involvement in Alzheimer disease. Neuropathol. Exp. Neurol. 58, 1125-1134 (1999).

4. Villain, N. et al. Regional dynamics of amyloid-beta deposition in healthy elderly, mild cognitive impairment and Alzheimer's disease: a voxelwise PiBPET longitudinal study. Brain 135, 2126-2139 (2012).

5. Thal, D. R., Rub, U., Orantes, M. \& Braak, H. Phases of A beta-deposition in the human brain and its relevance for the development of AD. Neurology $\mathbf{5 8}$ 1791-1800 (2002).

6. Benzinger, T. L. et al. Regional variability of imaging biomarkers in autosomal dominant Alzheimer's disease. Proc. Natl Acad. Sci. USA 110, E4502-4509 (2013).

7. Bateman, R. J. et al. Clinical and biomarker changes in dominantly inherited Alzheimer's disease. N. Engl. J. Med. 367, 795-804 (2012).

8. Blennow, K., Zetterberg, H. \& Fagan, A. M. Fluid biomarkers in Alzheimer disease. Cold Spring Harb. Perspect. Med. 2, a006221 (2012).

9. Fagan, A. M. et al. Cerebrospinal fluid tau and ptau(181) increase with cortical amyloid deposition in cognitively normal individuals: implications for future clinical trials of Alzheimer's disease. EMBO Mol. Med. 1, 371-380 (2009).

10. Mattsson, N. et al. Independent information from cerebrospinal fluid $\beta$-amyloid and florbetapir imaging in Alzheimer's disease. Brain 138, 772-783 (2014).

11. Morris, J. C. et al. APOE predicts amyloid-beta but not tau Alzheimer pathology in cognitively normal aging. Ann. Neurol. 67, 122-131 (2010).

12. Palmqvist, S., Mattsson, N., Hansson, O. \& Alzheimer's Disease Neuroimaging Initiative Cerebrospinal fluid analysis detects cerebral amyloid-beta accumulation earlier than positron emission tomography. Brain 139, 1226-1236 (2016).

13. Mattsson, N. et al. Predicting reduction of cerebrospinal fluid beta-amyloid 42 in cognitively healthy controls. JAMA Neurol. 72, 554-560 (2015).

14. Mattsson, N. et al. Emerging beta-amyloid pathology and accelerated cortical atrophy. JAMA Neurol. 71, 725-734 (2014).

15. Yeo, B. T. et al. The organization of the human cerebral cortex estimated by intrinsic functional connectivity. J. Neurophysiol. 106, 1125-1165 (2011).

16. Villemagne, V. L. et al. Longitudinal assessment of Abeta and cognition in aging and Alzheimer disease. Ann. Neurol. 69, 181-192 (2011).

17. Villeneuve, S. et al. Existing Pittsburgh Compound-B positron emission tomography thresholds are too high: statistical and pathological evaluation. Brain 138, 2020-2033 (2015)

18. Cho, H. et al. In vivo cortical spreading pattern of tau and amyloid in the Alzheimer disease spectrum. Ann. Neurol. 80, 247-258 (2016).

19. Braak, H. \& Braak, E. Neuropathological stageing of Alzheimer-related changes. Acta Neuropathol. 82, 239-259 (1991).

20. Andrews-Hanna, J. R., Smallwood, J. \& Spreng, R. N. The default network and self-generated thought: component processes, dynamic control, and clinical relevance. Ann. N. Y. Acad. Sci. 1316, 29-52 (2014).

21. Greicius, M. D. et al. Persistent default-mode network connectivity during light sedation. Hum. Brain Mapp. 29, 839-847 (2008).

22. Raichle, M. E. The brain's default mode network. Annu. Rev. Neurosci. 38, 433-447 (2015).

23. Buckner, R. L. et al. Molecular, structural, and functional characterization of Alzheimer's disease: evidence for a relationship between default activity, amyloid, and memory. J. Neurosci. 25, 7709-7717 (2005).

24. Sperling, R. A. et al. Amyloid deposition is associated with impaired default network function in older persons without dementia. Neuron 63, 178-188 (2009).

25. Myers, N. et al. Within-patient correspondence of amyloid-beta and intrinsic network connectivity in Alzheimer's disease. Brain 137, 2052-2064 (2014).

26. Sepulcre, J., Sabuncu, M. R., Becker, A., Sperling, R. \& Johnson, K. A. In vivo characterization of the early states of the amyloid-beta network. Brain 136, 2239-2252 (2013).

27. Cirrito, J. R. et al. Endocytosis is required for synaptic activity-dependent release of amyloid-beta in vivo. Neuron 58, 42-51 (2008).

28. Kamenetz, F. et al. APP processing and synaptic function. Neuron 37, 925-937 (2003).

29. $\mathrm{Li}, \mathrm{X}$. et al. Neuronal activity and secreted amyloid beta lead to altered amyloid beta precursor protein and presenilin 1 interactions. Neurobiol. Dis. 50, 127-134 (2013)

30. Lehmann, M. et al. Intrinsic connectivity networks in healthy subjects explain clinical variability in Alzheimer's disease. Proc. Natl Acad. Sci. USA 110, 11606-11611 (2013).

31. Buckner, R. L. et al. Cortical hubs revealed by intrinsic functional connectivity: mapping, assessment of stability, and relation to Alzheimer's disease. $J$. Neurosci. 29, 1860-1873 (2009). 
32. Elman, J. A. et al. Effects of beta-amyloid on resting state functional connectivity within and between networks reflect known patterns of regional vulnerability. Cereb. Cortex 26, 695-707 (2016).

33. Koch, K. et al. Disrupted intrinsic networks link amyloid-beta pathology and impaired cognition in prodromal Alzheimer's disease. Cereb. Cortex 25, 4678-4688 (2015).

34. Bero, A. W. et al. Neuronal activity regulates the regional vulnerability to amyloid-beta deposition. Nat. Neurosci. 14, 750-756 (2011).

35. Busche, M. A. et al. Critical role of soluble amyloid-beta for early hippocampal hyperactivity in a mouse model of Alzheimer's disease. Proc. Natl Acad. Sci. USA 109, 8740-8745 (2012).

36. Augutis, K. et al. Cerebrospinal fluid biomarkers of beta-amyloid metabolism in multiple sclerosis. Mult. Scler. 19, 543-552 (2013).

37. Mattsson, N. et al. Reduced cerebrospinal fluid BACE1 activity in multiple sclerosis. Mult. Scler. 15, 448-454 (2009).

38. van Westen, D. et al. Cerebral white matter lesions - associations with Abeta isoforms and amyloid PET. Sci. Rep. 6, 20709 (2016).

39. Scholl, M. et al. Low PiB PET retention in presence of pathologic CSF biomarkers in Arctic APP mutation carriers. Neurology 79, 229-236, (2012).

40. Vandenberghe, R., Adamczuk, K., Dupont, P., Laere, K. V. \& Chetelat, G. Amyloid PET in clinical practice: its place in the multidimensional space of Alzheimer's disease. Neuroimage Clin. 2, 497-511 (2013).

41. Sehlin, D. et al. Antibody-based PET imaging of amyloid beta in mouse models of Alzheimer's disease. Nat. Commun. 7, 10759 (2016).

42. Klunk, W. E. et al. The Centiloid Project: standardizing quantitative amyloid plaque estimation by PET. Alzheimers Dement. 11, 1-15.e4 (2015).

43. Bangen, K. J. et al. Cortical amyloid burden differences across empiricallyderived mild cognitive impairment subtypes and interaction with APOE varepsilon4 genotype. J. Alzheimers Dis. 52, 849-861 (2016).

44. Edmonds, E. C. et al. Susceptibility of the conventional criteria for mild cognitive impairment to false-positive diagnostic errors. Alzheimers Dement. 11, 415-424 (2015).

45. Edmonds, E. C. et al. Subjective cognitive complaints contribute to misdiagnosis of mild cognitive impairment. J. Int. Neuropsychol. Soc. 20, 836-847 (2014).

46. Folstein, M. F., Folstein, S. E. \& McHugh, P. R. "Mini-mental state”. A practical method for grading the cognitive state of patients for the clinician. J. Psychiatr. Res. 12, 189-198 (1975).

47. Morris, J. C. The Clinical Dementia Rating (CDR): current version and scoring rules. Neurology 43, 2412-2414 (1993).

48. Jack, C. R. Jr. et al. The Alzheimer's Disease Neuroimaging Initiative (ADNI): MRI methods. J. Magn. Reson. Imaging 27, 685-691 (2008).

49. Landau, S. M. et al. Measurement of longitudinal beta-amyloid change with 18F-florbetapir PET and standardized uptake value ratios. J. Nucl. Med. 56, 567-574 (2015)

50. Rasmussen, J. M. et al. Empirical derivation of the reference region for computing diagnostic sensitive (1)(8)fluorodeoxyglucose ratios in Alzheimer's disease based on the ADNI sample. Biochim. Biophys. Acta 1822, 457-466 (2012).

51. Rousset, O. G., Ma, Y. \& Evans, A. C. Correction for partial volume effects in PET: principle and validation. J. Nucl. Med. 39, 904-911 (1998).

52. Han, X. \& Fischl, B. Atlas renormalization for improved brain MR image segmentation across scanner platforms. IEEE Trans. Med. Imaging 26, 479-486 (2007).

53. Grabner, G., Janke, A. L., Budge, M. M., Smith, D., Pruessner, J., \& Collins, D. L. in Medical Image Computing and Computer-Assisted Intervention -- MICCAI 2006: 9th International Conference, Copenhagen, Denmark, October 1-6, 2006. Proceedings, Part II. (eds Larsen, R., Nielsen, M. \& Sporring J.) 58-66 (Berlin, Heidelberg, Springer, 2006).

54. Avants, B. B. et al. The Insight ToolKit image registration framework. Front. Neuroinform. 8, 44 (2014)

55. Thomas, B. A. et al. The importance of appropriate partial volume correction for PET quantification in Alzheimer's disease. Eur. J. Nucl. Med. Mol. Imaging 38, 1104-1119 (2011)

56. Olsson, A. et al. Simultaneous measurement of beta-amyloid(1-42), total tau, and phosphorylated tau (Thr181) in cerebrospinal fluid by the xMAP technology. Clin. Chem. 51, 336-345 (2005).

57. Shaw, L. M. et al. Cerebrospinal fluid biomarker signature in Alzheimer's disease neuroimaging initiative subjects. Ann. Neurol. 65, 403-413 (2009).

58. De Meyer, G. et al. Diagnosis-independent Alzheimer disease biomarker signature in cognitively normal elderly people. Arch. Neurol. 67, 949-956 (2010)

59. Weigand, S. D. et al. Transforming cerebrospinal fluid Abeta42 measures into calculated Pittsburgh Compound B units of brain Abeta amyloid. Alzheimers Dement. 7, 133-141 (2011).

60. Palmqvist, S. et al. Accuracy of brain amyloid detection in clinical practice using cerebrospinal fluid beta-amyloid 42: a cross-validation study against amyloid positron emission tomography. JAMA Neurol. 71, 1282-1289 (2014).
61. Toledo, J. B. et al. Nonlinear association between cerebrospinal fluid and florbetapir F-18 beta-amyloid measures across the spectrum of Alzheimer disease. JAMA Neurol. 72, 571-581 (2015).

62. Palmqvist, S. et al. Detailed comparison of amyloid PET and CSF biomarkers for identifying early Alzheimer's disease. Neurology 85, 1240-1249 (2015).

63. Jenkinson, M., Beckmann, C. F., Behrens, T. E. J., Woolrich, M. W. \& Smith, S. M. FSL. NeuroImage 62, 782-790 (2012).

64. Cox, R. W. AFNI: software for analysis and visualization of functional magnetic resonance neuroimages. Comput. Biomed. Res. 29, 162-173 (1996).

65. Avants, B. B. et al. The Insight ToolKit image registration framework. Front. Neuroinform. 8, 44 (2014).

66. Behzadi, Y., Restom, K., Liau, J. \& Liu, T. T. A component based noise correction method (CompCor) for BOLD and perfusion based fMRI. NeuroImage 37, 90-101 (2007).

67. Friston, K. J., Williams, S., Howard, R. \& Frackowiak, R. S. J. Movement-related effects in fMRI time-series. Magn. Reson. Med. 35, 346-55 (1996).

68. Power, J. D., Barnes, K. A., Snyder, A. Z., Schlaggar, B. L. \& Petersen, S. E. Spurious but systematic correlations in functional connectivity MRI networks arise from subject motion. NeuroImage 59, 2142-2154 (2012).

69. Friston, K. J., Williams, S., Howard, R., Frackowiak, R. S. \& Turner, R. Movement-related effects in fMRI time-series. Magn. Reson. Med. 35, 346-355 (1996).

70. He, H. \& Liu, T. T. A geometric view of global signal confounds in resting-state functional MRI. NeuroImage 59, 2339-2348 (2012).

71. Yeo, B. T. T. et al. The organization of the human cerebral cortex estimated by intrinsic functional connectivity. J. Neurophysiol. 106, 1125-1165 (2011).

72. Desikan, R. S. et al. An automated labeling system for subdividing the human cerebral cortex on MRI scans into gyral based regions of interest. NeuroImage 31, 968-980 (2006)

73. Zalesky, A., Fornito, A. \& Bullmore, E. T. Network-based statistic: identifying differences in brain networks. NeuroImage 53, 1197-1207 (2010).

74. Benjamini, Y. \& Hochberg, J. Controlling the false discovery rate: a practical and powerful approach to multiple testing. J. R. Stat. Soc. 57, 289-300 (1995).

\section{Acknowledgements}

The data used in preparation of this article were obtained from the ADNI database (adni. loni.usc.edu). As such, the investigators within the ADNI contributed to the design and implementation of ADNI and/or provided data but did not participate in analysis or writing of this report. A complete listing of ADNI investigators can be found at: http:// adni.loni.usc.edu/wp-content/uploads/how_to_apply/ADNI_Acknowledgement_List. pdf. Data from the Swedish BioFINDER study was used for this study. A complete list of BioFINDER members can be found can be found at http://biofinder.se/ the_biofinder_study_group/. We would also like to thank Ruben Smith and Gunnar Gouras for valuable input on the manuscript. Work at the authors' research centers was supported by the European Research Council, the Swedish Research Council, the Strategic Research Area MultiPark (Multidisciplinary Research in Parkinson's disease) at Lund University, the Swedish Brain Foundation, the Swedish Alzheimer Association, the Marianne and Marcus Wallenberg Foundation, the Skåne University Hospital Foundation, the Swedish federal government under the ALF agreement, the Knut and Alice Wallenberg Foundation and the Torsten Söderberg Foundation at the Royal Swedish Academy of Sciences. ADNI data collection and sharing for this project was funded by the ADNI (National Institutes of Health Grant U01 AG024904) and DOD ADNI (Department of Defense award number W81XWH-12-2-0012). ADNI is funded by the National Institute on Aging, the National Institute of Biomedical Imaging, and Bioengineering, and through generous contributions from the following: AbbVie, Alzheimer's Association; Alzheimer's Drug Discovery Foundation; Araclon Biotech; BioClinica, Inc.; Biogen; Bristol-Myers Squibb Company; CereSpir, Inc.; Cogstate; Eisai Inc.; Elan Pharmaceuticals, Inc.; Eli Lilly and Company; EuroImmun; F. Hoffmann-La Roche Ltd and its affiliated company Genentech, Inc.; Fujirebio; GE Healthcare; IXICO Ltd.; Janssen Alzheimer Immunotherapy Research \& Development, LLC.; Johnson \& Johnson Pharmaceutical Research \& Development LLC.; Lumosity; Lundbeck; Merck \& Co., Inc.; Meso Scale Diagnostics, LLC.; NeuroRx Research; Neurotrack Technologies; Novartis Pharmaceuticals Corporation; Pfizer Inc.; Piramal Imaging; Servier; Takeda Pharmaceutical Company; and Transition Therapeutics. The Canadian Institutes of Health Research is providing funds to support ADNI clinical sites in Canada. Private sector contributions are facilitated by the Foundation for the National Institutes of Health (www.fnih.org). The grantee organization is the Northern California Institute for Research and Education, and the study is coordinated by the Alzheimer's Therapeutic Research Institute at the University of Southern California. ADNI data are disseminated by the Laboratory for Neuro Imaging at the University of Southern California.

\section{Author contributions}

S.P. contributed to the study design and concept, interpreted data, performed the ROIbased statistical analyses, and drafted the manuscript. M.S. contributed to the study design and concept, interpreted data, and performed the voxel-based analyses. O.S 
processed all image data, interpreted data, and performed the connectivity analyses. N.M. contributed to the study design, interpreted data, and provided data on CSF converters. E.S. provided data on cognitively healthy elderly in BioFINDER. H.Z. and K.B. performed CSF analyses in BioFINDER and interpreted data. S.L. and W.J. contributed with ADNI florbetapir data and interpreted data. O.H. was P.I. for BioFINDER, was responsible for the study design and concept, and interpreted data. All co-authors have read and critically revised the manuscript.

\section{Additional information}

Supplementary Information accompanies this paper at doi:10.1038/s41467-017-01150-x.

Competing interests: S.P., M.S., O.S., N.M., and E.S. report no competing financial interests. K.B. has served as a consultant or at advisory boards for Alzheon, Eli Lilly,

Fujirebio Europe, I.B.L. International, Novartis, and Roche Diagnostics. K.B. and H.Z. are co-founders of Brain Biomarker Solutions in Gothenburg AB, a GU Venture-based platform company at the University of Gothenburg. S.L. has consulted for Biogen, Synarc, and Genentech. W.J. serves as a consultant to Bioclinica, Genentech, and Novartis

pharmaceuticals. O.H. has acquired research support (for the institution) from Roche, GE Healthcare, Biogen, AVID Radiopharmaceuticals, Fujirebio, and Euroimmun and in the past 2 years, he has received consultancy or speaker fees (paid to the institution) from Lilly, Roche, and Fujirebio. ${ }^{18}$ F-flutemetamol was generously provided by GE Healthcare in the BioFINDER study.
Reprints and permission information is available online at http://npg.nature.com/ reprintsandpermissions/

Publisher's note: Springer Nature remains neutral with regard to jurisdictional claims in published maps and institutional affiliations.

\section{(c) (i)}

Open Access This article is licensed under a Creative Commons Attribution 4.0 International License, which permits use, sharing, adaptation, distribution and reproduction in any medium or format, as long as you give appropriate credit to the original author(s) and the source, provide a link to the Creative Commons license, and indicate if changes were made. The images or other third party material in this article are included in the article's Creative Commons license, unless indicated otherwise in a credit line to the material. If material is not included in the article's Creative Commons license and your intended use is not permitted by statutory regulation or exceeds the permitted use, you will need to obtain permission directly from the copyright holder. To view a copy of this license, visit http://creativecommons.org/ licenses/by/4.0/.

(c) The Author(s) 2017 